\title{
On Optimal Beamforming Design for Downlink MISO NOMA Systems
}

\author{
Jianyue Zhu, Jiaheng Wang, Yongming Huang, Keivan Navaie, Zhiguo Ding, Luxi Yang
}

\begin{abstract}
This work focuses on the beamforming design for downlink multiple-input single-output (MISO) nonorthogonal multiple access (NOMA) systems. The beamforming vectors are designed by solving a total transmission power minimization (TPM) problem with quality-of-service (QoS) constraints. In order to solve the proposed nonconvex optimization problem, we provide an efficient method using semidefinite relaxation. Moreover, for the first time, we characterize the optimal beamforming in a closed form with quasi-degradation condition, which is proven to achieve the same performance as dirtypaper coding (DPC). For the special case with two users, we further show that the original nonconvex TPM problem can be equivalently transferred into a convex optimization problem and easily solved via standard optimization tools. In addition, the optimal beamforming is also characterized in a closed form and we show that it achieves the same performance as the DPC. In the simulation, we show that our proposed optimal NOMA beamforming outperforms OMA schemes and can even achieve the same performance as DPC. Our solutions dramatically simplifies the problem of beamforming design in the downlink MISO NOMA systems and improve the system performance.
\end{abstract}

Index Terms-Nonorthogonal multiple access, transmission power minimization, multiple-input single-output, quasidegradation, quality-of-service

\section{INTRODUCTION}

The beyond fifth generation (B5G) wireless networks impose high data rate requirements with the exponential growth of data traffic for wireless communication [1]. A lot of potential technologies, e.g., massive multiple-input multipleoutput (MIMO) [2] , small cells [3], and device to device communication [4], [5] are introduced to the B5G communication systems to deal with the demand of enormous data traffic. Particularly, being able to provide the flexibility and degrees of freedom to achieve higher spectral efficiency, multiantenna transmission has been widely regarded as the key technology for the upcoming $\mathrm{B} 5 \mathrm{G}$ communication systems.

This work was supported by the National Key R\&D Program of China under Grant 2018YFB1800801, the National Natural Science Foundation of China under Grant 61720106003, 61971130, 61571107, 61711540305, and 61901107, the Research Project of Jiangsu Province under Grant BE2018121, BK20160069, and BK20190337, the UK EPSRC under grant number EP/P009719/2, the H2020-MSCA-RISE-2015 under grant number 690750, the Scientific Research Foundation of Graduate School of Southeast University under Grant YBJJ1816, and the Scholarship from China Scholarship Council under Grant 201806090065. (Corresponding author: Yongming Huang.)

J. Zhu, J. Wang, Y. Huang, and L. Yang are with the National Mobile Communications Research Laboratory, Southeast University, Nanjing, China. (email: \{zhujy, jhwang, huangym, lxyang\}@seu.edu.cn).

K. Navaie is with the School of Computing and Communications, Lancaster University, Lancaster LA1 4WA, United Kingdom (email: k.navaie@lancaster.ac.uk).

Z. Ding is with the School of Electrical and Electronic Engineering, Manchester University, Manchester, UK (email: zhiguo.ding@manchester.ac.uk).
In conventional multiantenna systems, e.g., [6], [7], orthogonal multiple access (OMA) schemes are often exploited. In these OMA techniques, the resources (time, frequency, or code) are allocated to each user exclusively and hence multiuser interference can be avoided. However, the limited spectral resources cannot be fully exploited by using these traditional resource allocation strategies.

Zero-forcing beamforming (ZFBF) is another widely used downlink precoding scheme. The ZFBF scheme uses the spatial degrees of freedom for interference avoidance [8], [9]. Specifically, in ZFBF strategy, the users transmit data in the null space of other users' channels and hence the multi-user interference is mitigated. Nevertheless, the ZFBF strategy is efficient only in cases where the BS has no less antennas than the receivers. In addition, dirty-paper coding (DPC) is also widely used in the literature. Suppose the transmitter has perfect channel state information, some works, e.g., [10], [11], showed that the capacity region can be achieved by using DPC. However, practically, considering the prohibitively high complexity, the scheme of DPC is difficult to be implemented.

To overcome the shortcomings of OMA, ZF or DPC, nonorthogonal multiple access (NOMA) was proposed, which can efficiently use the limited spectral resources [12], [13]. Specifically, in the NOMA systems, multiple users are able to share the same resources (time, frequency, code, or spatial). In the literature, there are a lot of works focusing on the technology of NOMA. For example, in [14], in order to achieve low latency for IoT, the authors applied NOMA in short-packet communication. [15] determined the optimal power allocation and redundancy rate to maximize the secrecy rate for the strong user in NOMA systems.

Since its lower complexity than DPC, NOMA based multiantenna transmission has attracted a lot of research interests. In [16], the authors described that NOMA based MIMO technologies have significant potential in improving spectral efficiency. [17] proposed a MIMO NOMA framework, where both of the downlink and uplink transmissions were studied.The authors in [18] studied the joint design of beamforming and power allocation for multi-cell multiuser MIMO NOMA. The authors in [19] studied a new secrecy beamforming scheme for multi-input single-output (MISO) NOMA systems. [20] also focused on secure performance of MISO NOMA and a novel beamforming design by using the optimal power allocation was proposed.

Because of the nonorthogonality of users, resource allocation is a key problem in the NOMA systems, which is challenging to solve [21], [22]. Nevertheless, in [23], the authors exhibited that single-input single-output (SISO) NOMA 
systems outperform conventional SISO OMA systems in terms of spectral efficiency, fairness, and energy efficiency. However, most existing works on NOMA resource allocation focused on SISO NOMA systems but the results cannot be directly applied to MISO NOMA systems. In fact, the resource allocation problem for MISO NOMA systems is a joint beamforming and power optimization problem. Compared to the SISO NOMA systems, the resource allocation for MISO NOMA systems is more difficult to solve.

In the literature, some works investigated the resource allocation problem in the downlink MISO NOMA systems. Specifically, [24] studied the NOMA beamforming design for maximizing all the strong users' sum rate. The NOMA beamforming design for maximizing the sum rate of all users was studied in [25]-[27]. For example, [25] investigated the beamforming design for sum rate maximization with quality of service (QoS) constraints. By employing the method of successive convex approximation (SCA), a suboptimal solution for this formulated optimization problem was obtained in [25]. In addition, in [25], the optimality of this obtained solution compared to the upper bound, DPC, is still unknown. However, in this work, we not only provide the closed form optimal solution but also proves that MISO NOMA can achieve the same performance as DPC.

It is worthy pointing out that energy consuming is another important performance of wireless communication systems. In this work, we investigate the design of NOMA beamforming for minimizing total transmission power with each user's QoS constraint. There are some relative works also focusing on beamforming design for minimizing total transmission power with QoS constraints in the MISO NOMA systems. In [28], the authors exploited the SCA method to solve the formulated optimization problem and provided the suboptimal solution. In [29], the authors only considered the special case with two users and proposed an iterative algorithm to find the beamforming solution, which is suboptimal and complex. [30] investigated the beamforming design for multiple users case, where the users are classified into multiple groups. However, in each group, the authors considered only two users and found the optimal beamforming via search algorithm with high complexity.

In addition, also for minimizing total transmission power with QoS constraints in the MISO NOMA systems, [11], [31] found the optimal beamforming vectors by studying the dual problem of the formulated optimization problem. The beamforming vectors were even shown in closed form with quasi-degradation condition. However, both of the works considered limited number of users, i.e., two users (in one group). To the best of our knowledge, there is no work providing the optimal beamforming solution for multiple users case.

In this paper, we study the optimal beamforming solution for minimizing transmission power of all the users with QoS constraints in the MISO NOMA systems. The contributions are summarized as follows:

- We consider minimizing total transmission power of all users with QoS constraints in the downlink MISO NOMA systems.
- By exploiting semidefinite relaxation (SDR), we provide an efficient method to solve the formulated nonconvex problem.

- We introduce the concept of quasi-degradation for multiple users case, which is used to identify the gap between the optimal performance of NOMA and that of DPC.

- Then, with quasi-degradation condition, we characterize the optimal beamforming solution to the formulated optimization problem in closed form, which is proven to achieve the same performance as DPC.

- Moreover, we provide the channel conditions to be quasidegraded for multiple users.

- In the special case with two users, we equivalently transform the original nonconvex problem into a convex one, which can be efficiently solved by using semidefinite programming (SDP).

- Furthermore, for two users case, we provide the closed form beamforming solution, which is proved to achieve the same performance as DPC. Compared with [11], [31], we derive an alternative and easier way to achieve the optimal closed form beamforming.

The rest of the paper is organized as follows. Section II describes the MISO NOMA system model and the formulated problem. Section III provides the solution to the formulated problem and investigates the optimal MISO NOMA beamforming with quasi-degradation condition. Section IV studies the optimal beamforming in the special case of two users. The performance of the proposed beamforming solution is shown in Section V via simulation. Finally, Section VI concludes this paper.

Notations: We respectively use lower case letters and boldface capital to denote vectors and matrices. $\boldsymbol{a}^{H}$ denotes the Hermitian transpose vector $\boldsymbol{a} ; \boldsymbol{A} \succeq \mathbf{0}$ indicates that $\boldsymbol{A}$ is a positive semidefinite matrix; $\operatorname{Rank}(\boldsymbol{A})$ and $\operatorname{Tr}(\boldsymbol{A})$ are respectively the rank and trace of matrix $\boldsymbol{A} ;|$.$| and \|$. respectively denote the absolute value of a complex scalar and the Euclidean vector norm; $\mathbb{C}^{T}$ denotes the set of complex numbers; $\boldsymbol{I}_{N}$ is the $N \times N$ identity matrix.

\section{System Model and Problem Formulation}

We consider a downlink NOMA system in which a base station (BS) with $T$ antennas serves $N$ single-antenna users. $s_{i}$ is the message to be received by user $i\left(\mathrm{UE}_{i}\right)$ with $E\left[\left|s_{i}\right|^{2}\right]=1$ and $\mathbf{w}_{i} \in \mathbb{C}^{T}$ be $\mathrm{UE}_{i}$ 's complex beamforming vector. In the NOMA systems, the BS transmits the superposition coding and hence each $\mathrm{UE}_{k}$ receives the signal

$$
y_{k}=\sum_{i=1}^{N} \mathbf{h}_{k}^{H} \boldsymbol{w}_{i} s_{i}+n_{k}, k=1, \cdots, N,
$$

where $\mathbf{h}_{k}=d_{k}^{-\alpha} \mathbf{g}_{k} \in \mathbb{C}^{T}$ (column vector) contains the channel coefficients from the $\mathrm{BS}$ to $\mathrm{UE}_{k}, d_{k}$ is the distance between $\mathrm{UE}_{k}$ and the BS, $\alpha$ is the exponent of path loss, $\mathrm{g}_{k}$ follows a Rayleigh distribution, and $n_{k} \sim \mathcal{C N}\left(0, \sigma^{2}\right)$ is the additive white Gaussian noise (AWGN).

In NOMA systems, each user employs successive interference cancellation (SIC) to decode their own signals. Without 
loss of generality, the order of users' channel gains is given by

$$
\left\|\mathbf{h}_{1}\right\|_{2}>\left\|\mathbf{h}_{2}\right\|_{2}>\cdots>\left\|\mathbf{h}_{N}\right\|_{2},
$$

i.e., $\mathrm{UE}_{1}$ and $\mathrm{UE}_{N}$ are respectively the strongest and weakest users. In the SISO NOMA systems, the optimal signal decoding order is same as the ascending order of the channel gains. However, in the MISO NOMA systems, it is difficult to know the optimal decoding order. Actually, the optimal decoding order is determined by the product of channel gain and beamforming vector [32]. Note that, in this paper, our focus is not the optimal decoding order and we still assume the decoding order as the ascending order of the channel gains. This also reduces the complexity of beamforming design. Therefore, $\mathrm{UE}_{k}$ can decode signals of $\mathrm{UE}_{n}$ for $n>k$ and remove them from its own signal, but treats the signals from $\mathrm{UE}_{n}$ for $n<k$ as interference. The achievable rate of $\mathrm{UE}_{k}$ for $k=1,2, \cdots, N$ using SIC is therefore

$$
R_{k}=\log \left(1+\min _{n \in\{1, \cdots, k\}} \operatorname{SINR}_{n, k}\right),
$$

where

$$
\operatorname{SINR}_{n, k}=\frac{\left|\mathbf{h}_{n}^{H} \boldsymbol{w}_{k}\right|^{2}}{\sum_{j=1}^{k-1}\left|\mathbf{h}_{n}^{H} \boldsymbol{w}_{j}\right|^{2}+\sigma^{2}},
$$

denotes the signal interference noise ratio (SINR) of $\mathrm{UE}_{n}$ to decode $\mathrm{UE}_{k}$ for $k=1, \cdots, N, n=1, \cdots, k$.

The performance of the MISO NOMA scheme relies on the design of beamforming vectors. In this work, we investigate the optimization of beamforming vectors for NOMA systems and assume that the perfect channel state information (CSI) is available at all nodes. We consider the total BS transmission power minimization (TPM) problem subject to guaranteeing the target rate for each user, which is formulated as follows

$$
\begin{aligned}
P_{\text {NOMA }}= & \min _{\left\{\boldsymbol{w}_{k}\right\}} \sum_{k=1}^{N}\left\|\boldsymbol{w}_{k}\right\|_{2}^{2}, \\
& \text { s.t. } R_{k} \geq R_{k}^{\min }, k=1,2, \cdots, N,
\end{aligned}
$$

where $R_{k}^{\min }$ represents the QoS threshold of $\mathrm{UE}_{k}$. The similar problems have been studied in [11], [28]-[31], whereas the optimal beamforming was only found for limited number of users, i.e., two users, but it is unknown for the general multiple users case.

In fact, the optimization of beamforming vectors for TPM is a difficult nonconvex problem. To the best of the authors' knowledge, the optimal beamforming for TPM for multiple users (more than two users) has not been achieved in the existing literature. In this paper, we first exploit the SDR to find the solution to (5). Then, we focus on the quasidegradation condition, which was firstly defined in [11] for two users and used to describe the gap between the optimal performance of NOMA and that of DPC. For multiple users, we investigate the optimal beamforming when the channels of users are quasi-degraded. In addition, in the special case with two users, compared to [11], we provide an alternative way to obtain the optimal beamforming. Specifically, we transfer the original nonconvex problem into an equivalent problem, which is convex and can be solved easily via SDP. Furthermore, we characterize the optimal beamforming in closed form with the quasi-degradation condition. Our results will dramatically simplify the problem of beamforming design in the downlink MISO NOMA systems and improve the system performance.

\section{BEAMFORMING DESIGN FOR TPM}

In this section, we study beamforming design for minimizing transmission power with multiple users. The similar problems have also been considered in [11], [28]-[31], while [11], [29]-[31] only considered the special case with two users and in [28], the authors proposed the suboptimal beamforming via successive approximations. The TPM problem, in problem (5), is indeed a nonconvex problem, which is difficult to find its solution. In this section, we firstly find the solution using semidefinite relaxation (SDR). Then, we will provide the optimal beamforming vectors in closed form for the cases where the channels of users are quasi-degraded. The definition of quasi-degradation was firstly proposed in [11] for two users. In this work, we further introduce this concept for multiple users.

\section{A. Beamforming design via SDR}

Here, we will use the SDR approach to solve problem (5). Firstly, we introduce the following variable transformation: $\boldsymbol{Q}_{k}=\boldsymbol{w}_{k} \boldsymbol{w}_{k}^{H}$. Therefore,

$$
\operatorname{SINR}_{n, k}=\frac{\mathbf{h}_{n}^{H} \boldsymbol{Q}_{k} \mathbf{h}_{n}}{\sum_{j=1}^{k-1} \mathbf{h}_{n}^{H} \boldsymbol{Q}_{j} \mathbf{h}_{n}+\sigma^{2}},
$$

for $k=1, \cdots, N, n=1, \cdots, k$. With $A_{k}^{\min }=2^{R_{k}^{\min }}-1$, the original TPM problem (5) is rewritten as

$$
\begin{aligned}
\min _{\left\{\boldsymbol{Q}_{k} \succeq \mathbf{0}\right\}} & \sum_{k=1}^{N} \operatorname{Tr}\left(\boldsymbol{Q}_{k}\right) \\
\text { s.t. } & \mathbf{h}_{n}^{H} \boldsymbol{Q}_{k} \mathbf{h}_{n}-A_{k}^{\min }\left(\sum_{j=1}^{k-1} \mathbf{h}_{n}^{H} \boldsymbol{Q}_{j} \mathbf{h}_{n}+\sigma^{2}\right) \geq 0 \\
& k=1, \cdots, N, n=1, \cdots, k \\
& \quad \operatorname{Rank}\left(\boldsymbol{Q}_{k}\right) \leq 1, k=1, \cdots, N .
\end{aligned}
$$

Problem (8) is still a nonconvex problem due to the rank constraints (10). We first consider the optimization problem (8) without the rank constraints (10), i.e.,

$$
\begin{aligned}
& \min _{\left\{\boldsymbol{Q}_{k} \succeq \mathbf{0}\right\}} \sum_{k=1}^{N} \operatorname{Tr}\left(\boldsymbol{Q}_{k}\right), \\
& \text { s.t. } \mathbf{h}_{n}^{H} \boldsymbol{Q}_{k} \mathbf{h}_{n}-A_{k}^{\min }\left(\sum_{j=1}^{k-1} \mathbf{h}_{n}^{H} \boldsymbol{Q}_{j} \mathbf{h}_{n}+\sigma^{2}\right) \geq 0, \\
& \quad k=1, \cdots, N, n=1, \cdots, k .
\end{aligned}
$$

One can easily find that problem (11) is a standard SDP, which is efficiently solved by using convex optimization techniques, e.g., CVX. However, the solution to problem (11) can not always satisfy the rank constraint (10). In general, if we obtain a set of rank-one matrix solutions to the relaxed problem 
in (11), then these matrices are also the optimal solutions to problem (8). Otherwise, we can use the randomization technique [33] to produce a set of rank-one solutions. After obtaining the solution: $\left\{\boldsymbol{Q}_{k}\right\}_{k=1}^{N}$, the beamforming vectors can be obtained by using the singular value decomposition (SVD) of $\boldsymbol{Q}_{k}$.

\section{B. The optimal beamforming in closed form with quasi- degraded channels}

In this subsection, the closed form beamforming solution is characterized when the channels of users are quasi-degraded. The definition of quasi-degradation was firstly proposed in [11], [31] for the special case with two users. In this paper, we will apply this concept to the general multiple users case in the downlink MISO NOMA systems.

In order to introduce the definition of quasi-degradation for multiple users, we propose the DPC transmission scheme. Above all, in order to describe DPC scheme, a encoding order $(N, \cdots, 1)$ is assumed at the $\mathrm{BS}$, which is consistent with the NOMA decoding order. Specifically, for $\mathrm{UE}_{k}$, the BS first encodes the information intended to $\mathrm{UE}_{l}$ for $l>k$, and then encodes the information intended to $\mathrm{UE}_{k}$ by pre-subtracting the information of $\mathrm{UE}_{l}$ for $l=k+1, \cdots, N$. Therefore, the achievable rate of $\mathrm{UE}_{k}$ is:

$$
C_{k}=\log \left(1+\frac{\mathbf{h}_{k}^{H} \boldsymbol{w}_{k} \boldsymbol{w}_{k}^{H} \mathbf{h}_{k}}{\sigma^{2}+\sum_{j=1}^{k-1} \mathbf{h}_{k}^{H} \boldsymbol{w}_{j} \boldsymbol{w}_{j}^{H} \mathbf{h}_{k}}\right),
$$

and the problem of beamforming design for TPM with QoS constraint is formulated as

$$
\begin{aligned}
P_{D P C}= & \min _{\left\{\boldsymbol{w}_{k}\right\}} \sum_{k=1}^{N}\left\|\boldsymbol{w}_{k}\right\|_{2}^{2}, \\
& \text { s.t. } C_{k} \geq R_{k}^{\min }, k=1, \cdots, N .
\end{aligned}
$$

The QoS constraints, (15), is in fact:

$$
\begin{aligned}
& \sum_{j=1}^{k-1} \frac{1}{\sigma^{2}} \mathbf{h}_{k}^{H} \boldsymbol{w}_{j} \boldsymbol{w}_{j}^{H} \mathbf{h}_{k}+1 \\
\leq & \frac{1}{\sigma^{2} A_{k}^{\min }} \mathbf{h}_{k}^{H} \boldsymbol{w}_{k} \boldsymbol{w}_{k}^{H} \mathbf{h}_{k}, k=1, \cdots, N .
\end{aligned}
$$

The optimal solution to problem (14) is obtained by using Lemma 1 and Proposition 1 presented in the following.

In general, the optimal solution to problem (5) is difficult to obtain. Here, however, by introducing the following definition, we can provide the optimal beamforming in closed form.

Definition 1. Assume a decoding order $(N, \cdots, 1)$ of NOMA and a encoding order $(N, \cdots, 1)$ of DPC, then the broadcast channels $\left\{\mathbf{h}_{k}\right\}_{k=1}^{N}$ are quasi-degraded with respect to the target QoS thresholds $\left\{R_{k}^{\min }\right\}_{k=1}^{N}$ if and only if the minimum total transmission power of MISO NOMA is equal to that of MISO DPC, i.e., $P_{\text {NOMA }}=P_{D P C}$.

Remark 1. It has been shown in [10] that the capacity region can be achieved by using DPC and hence DPC is commonly used as a benchmark. Definition 1 is used to identify the gap between the optimal performance of NOMA and that of DPC.
Specifically, if the channels of users are quasi-degraded, the optimal performance achieved by NOMA is same as DPC.

In order to obtain the closed form beamforming with quasidegraded channels in the MISO NOMA systems, we introduce the following Lemma.

Lemma 1. If $\left\{\boldsymbol{w}_{k}^{* N O M A}\right\}_{k=1}^{N}$ is the optimal solution to problem (5), and the broadcast channels $\left\{\mathbf{h}_{k}\right\}_{k=1}^{N}$ are quasidegraded, there exists the optimal solution $\left\{\boldsymbol{w}_{k}^{* D P C}\right\}_{k=1}^{N}$ to problem (14), such that

$$
\left\{\boldsymbol{w}_{k}^{* D P C}\right\}_{k=1}^{N}=\left\{\boldsymbol{w}_{k}^{* N O M A}\right\}_{k=1}^{N}
$$

Proof. Please refer to Appendix A.

According to Lemma 1, with quasi-degradation condition, the optimal solution to (5) can be obtained by solving the problem (14). Therefore, we can focus on solving problem (14).

However, problem (14) is also nonconvex and can not be directly solved. In order to characterize the hidden convexity of problem (14), we present the following Lemma.

Lemma 2. The inner product of $\left\{\mathbf{h}_{k}^{H} \mathbf{w}_{k}\right\}_{k=1}^{N}$ can be transferred to a real value without loss of optimality in (14).

Proof. Please refer to Appendix B.

Using Lemma 2, we then find out the hidden convexity of problem (14) in the following Proposition.

Proposition 1. The problem (14) can be written as

$$
\begin{aligned}
& \min _{\left\{\boldsymbol{w}_{k}\right\}} \sum_{k=1}^{N}\left\|\boldsymbol{w}_{k}\right\|_{2}^{2}, \\
& \text { s.t. } \sqrt{\sum_{j=1}^{k-1} 1 / \sigma^{2} \mathbf{h}_{k}^{H} \boldsymbol{w}_{j} \boldsymbol{w}_{j}^{H} \mathbf{h}_{k}+1} \leq \frac{1}{\sigma \sqrt{A_{k}^{\min }}} \mathbf{h}_{k}^{H} \boldsymbol{w}_{k}, \\
& \quad \text { for } k=1, \cdots, N
\end{aligned}
$$

where $A_{k}^{\min }=2^{R_{k}^{\min }}-1, k=1, \cdots, N$ and (18) is a convex optimization problem.

Proof. Please refer to Appendix C.

Corollary 1. The convex form of problem (18) has the same Lagrangian as the original form of problem (14).

Proof. Please refer to Appendix D.

Consequently, according to Proposition 1, one can easily find the optimal solution to problem (14) via standard optimization tools, e.g., CVX. Moreover, from Corollary 1, the optimal beamforming can even be characterized in closed form by exploiting the Lagrangian of problem (14). Therefore, with the condition of quasi-degradation, we can obtain the optimal beamforming solution to problem (5) by solving problem (14) (or (18)). To the best of the authors' knowledge, there is no work finding out the optimal beamforming in the downlink MISO NOMA systems. In the following Proposition, with quasi-degradation condition, we will characterize the optimal beamforming vectors in closed form. 
Proposition 2. Suppose that the channels of users are quasidegraded, then the optimal solution to problem (5) is $\boldsymbol{w}_{k}^{*}=$ $\sqrt{p_{k}} \tilde{\boldsymbol{w}}_{k}, k=1, \cdots, N$, where

$$
\tilde{\boldsymbol{w}}_{k}=\frac{\left(\boldsymbol{I}_{T}+\sum_{j=k+1}^{N} \frac{\lambda_{j}}{\sigma^{2}} \mathbf{h}_{j} \mathbf{h}_{j}^{H}\right)^{-1} \mathbf{h}_{k}}{\left|\left(\boldsymbol{I}_{T}+\sum_{j=k+1}^{N} \frac{\lambda_{j}}{\sigma^{2}} \mathbf{h}_{j} \mathbf{h}_{j}^{H}\right)^{-1} \mathbf{h}_{k}\right|}
$$

is the normalized directions for $U E_{k}$ with

$$
\lambda_{k}=\frac{\sigma^{2} A_{k}^{\min }}{\mathbf{h}_{k}^{H}\left(\boldsymbol{I}_{T}+\sum_{j=k+1}^{N} \frac{\lambda_{j}}{\sigma^{2}} \mathbf{h}_{j} \mathbf{h}_{j}^{H}\right)^{-1} \mathbf{h}_{k}}, k=1, \cdots, N-1,
$$

$\lambda_{N}=\frac{\sigma^{2} A_{N}^{\min }}{\mathbf{h}_{N}^{H} \mathbf{h}_{N}}$,

and

$$
\begin{aligned}
p_{1} & =\frac{A_{1}^{\min } \sigma^{2}}{\mathbf{h}_{1}^{H} \tilde{\boldsymbol{w}}_{1} \tilde{\boldsymbol{w}}_{1}{ }^{H} \mathbf{h}_{1}}, \\
p_{k} & =\frac{A_{k}^{\min } \mathbf{h}_{k}^{H}\left(\sum_{j=1}^{k-1} p_{j} \tilde{\boldsymbol{w}}_{j} \tilde{\boldsymbol{w}}_{j}{ }^{H}\right) \mathbf{h}_{k}+A_{k}^{\min } \sigma^{2}}{\mathbf{h}_{k}^{H} \tilde{\boldsymbol{w}}_{k} \tilde{\boldsymbol{w}}_{k}{ }^{H} \mathbf{h}_{k}}, \\
k & =2, \cdots, N,
\end{aligned}
$$

are the optimal transmit powers.

Proof. Please refer to Appendix E.

Corollary 2. Given $\left\|\mathbf{h}_{1}\right\|_{2}>\cdots>\left\|\mathbf{h}_{N}\right\|_{2}$, the optimal transmit powers always satisfy $p_{1} \leq \cdots \leq p_{N}$ if

$$
C 1: A_{k+1}^{\min }+A_{k+1}^{\min } A_{k}^{\min } \geq A_{k}^{\min } .
$$

Proof. Please refer to Appendix F.

Therefore, for multiple users, when the channels are quasidegraded, the optimal beamforming for TPM can be characterized by Proposition 2. In addition, Corollary 2 shows that, with reasonable value of QoS thresholds, the optimal allocated power to the weak users is higher. This contributes into fairness of resource allocation in the MISO NOMA systems.

With the optimal beamforming proposed in Proposition 2, Proposition 3 provides an explicit necessary and sufficient condition for quasi-degraded channels with multiple users. Compared to [11], [31], where the quasi-degradation condition was for two users, but we provide the quasi-degradation condition for multiple users.

Proposition 3. The broadcast channels $\left\{\mathbf{h}_{k}\right\}_{k=1}^{N}$ are quasidegraded with respect to $\left\{A_{k}^{\min }\right\}_{k=1}^{N}$, if and only if

$$
\begin{gathered}
C 2: \mathbf{h}_{n}^{H} \boldsymbol{w}_{k}^{*} \boldsymbol{w}_{k}^{* H} \mathbf{h}_{n}-A_{k}^{\min }\left(\sum_{j=1}^{k-1} \mathbf{h}_{n}^{H} \boldsymbol{w}_{j}^{*} \boldsymbol{w}_{j}^{* H} \mathbf{h}_{n}+\sigma^{2}\right) \geq 0 \\
\text { for } k=2, \cdots, N, n=1, \cdots, k-1,
\end{gathered}
$$

where $\boldsymbol{w}_{k}^{*}$ is given in Proposition 2.

Proof. Please refer to Appendix G.
With perfect CSI available at the transmitter, it has been shown that the best performance can be achieved by using DPC [10], [11]. However, DPC is hard to be used practically in communication systems. Proposition 3 shows that, with the quasi-degradation condition, the proposed downlink NOMA transmission scheme can simultaneously yield performance of the performance region of MISO system.

In the general case of multiple users in the downlink MISO NOMA systems, since the number of constraints is very large, we only provide the optimal beamforming when the channels are quasi-degraded. In the following section, we the optimal solution for a system with two users is provided.

\section{Optimal BeAmForming For TPM WITH Two UsERS}

In this section, we focus on the special case that there are two users performing NOMA in a MISO system. Note that the additional complexity of SIC is proportional to the number of users. Therefore, the case with two users is often adopted in some works [11], [29]. In this case, the TPM problem is

$$
\begin{aligned}
& \min _{\left\{\boldsymbol{w}_{1}, \boldsymbol{w}_{2}\right\}}\left\|\boldsymbol{w}_{1}\right\|_{2}^{2}+\left\|\boldsymbol{w}_{2}\right\|_{2}^{2}, \\
& \text { s.t. } R_{1} \geq R_{1}^{\mathrm{min}}, R_{2} \geq R_{2}^{\mathrm{min}},
\end{aligned}
$$

where

$$
\begin{aligned}
& R_{1}=\log \left(1+\frac{\mathbf{h}_{1}^{H} \boldsymbol{w}_{1} \boldsymbol{w}_{1}^{H} \mathbf{h}_{1}}{\sigma^{2}}\right), \\
& R_{2}=\min \left\{\log \left(1+\operatorname{SINR}_{1,2}\right), \log \left(1+\operatorname{SINR}_{2,2}\right)\right\},
\end{aligned}
$$

and

$$
\begin{aligned}
\operatorname{SINR}_{1,2} & =\frac{\mathbf{h}_{1}^{H} \boldsymbol{w}_{2} \boldsymbol{w}_{2}^{H} \mathbf{h}_{1}}{\sigma^{2}+\mathbf{h}_{1}^{H} \boldsymbol{w}_{1} \boldsymbol{w}_{1}^{H} \mathbf{h}_{1}}, \\
\operatorname{SINR}_{2,2} & =\frac{\mathbf{h}_{2}^{H} \boldsymbol{w}_{2} \boldsymbol{w}_{2}^{H} \mathbf{h}_{2}}{\sigma^{2}+\mathbf{h}_{2}^{H} \boldsymbol{w}_{1} \boldsymbol{w}_{1}^{H} \mathbf{h}_{2}} .
\end{aligned}
$$

This problem was also studied in [11], [29]-[31], however, [29], [30] only gave the suboptimal solution. In this section, we provide the optimal beamforming solution, which is also characterized in closed form. In addition, compared with [11], [31], we directly deal with the original optimization problem instead of the dual problem and propose an alternative approach to solve (27).

\section{A. Optimal beamforming via SDR}

Although the original TPM problem (27) is nonconvex, we obtain its optimal solution by considering its equivalent problem using variable transformation: $\boldsymbol{Q}_{k}=\boldsymbol{w}_{k} \boldsymbol{w}_{k}^{H}, k=1,2$. 
Setting $A_{k}^{\min }=2^{R_{k}^{\min }}-1, k=1,2$, the original problem, (27), is transferred into

$$
\begin{aligned}
\min _{\left\{\boldsymbol{Q}_{1} \succeq \mathbf{0}, \boldsymbol{Q}_{2} \succeq \mathbf{0}\right\}} & \operatorname{Tr}\left(\boldsymbol{Q}_{1}+\boldsymbol{Q}_{2}\right), \\
\text { s.t. } & A_{1}^{\min } \sigma^{2}-\mathbf{h}_{1}^{H} \boldsymbol{Q}_{1} \mathbf{h}_{1} \leq 0, \\
& \frac{1}{\sigma^{2}} \mathbf{h}_{1}^{H} \boldsymbol{Q}_{1} \mathbf{h}_{1}-\frac{1}{\sigma^{2} A_{2}^{\min }} \mathbf{h}_{1}^{H} \boldsymbol{Q}_{2} \mathbf{h}_{1}+1 \leq 0, \\
& \frac{1}{\sigma^{2}} \mathbf{h}_{2}^{H} \boldsymbol{Q}_{1} \mathbf{h}_{2}-\frac{1}{\sigma^{2} A_{2}^{\min }} \mathbf{h}_{2}^{H} \boldsymbol{Q}_{2} \mathbf{h}_{2}+1 \leq 0, \\
& \operatorname{Rank}\left(\boldsymbol{Q}_{k}\right) \leq 1, k=1,2,
\end{aligned}
$$

which is also a nonconvex problem because of the rank constraint in (37). Here, we set aside the rank constraints, the SDR of problem (33) is

$$
\begin{aligned}
\min _{\left\{\boldsymbol{Q}_{1} \succeq \mathbf{0}, \boldsymbol{Q}_{2} \succeq \mathbf{0}\right\}} & \operatorname{Tr}\left(\boldsymbol{Q}_{1}+\boldsymbol{Q}_{2}\right), \\
\text { s.t. } & A_{1}^{\min } \sigma^{2}-\mathbf{h}_{1}^{H} \boldsymbol{Q}_{1} \mathbf{h}_{1} \leq 0, \\
& \frac{1}{\sigma^{2}} \mathbf{h}_{1}^{H} \boldsymbol{Q}_{1} \mathbf{h}_{1}-\frac{1}{\sigma^{2} A_{2}^{\min }} \mathbf{h}_{1}^{H} \boldsymbol{Q}_{2} \mathbf{h}_{1}+1 \leq 0, \\
& \frac{1}{\sigma^{2}} \mathbf{h}_{2}^{H} \boldsymbol{Q}_{1} \mathbf{h}_{2}-\frac{1}{\sigma^{2} A_{2}^{\min }} \mathbf{h}_{2}^{H} \boldsymbol{Q}_{2} \mathbf{h}_{2}+1 \leq 0 .
\end{aligned}
$$

Note that problem (38) is a standard SDP. Hence, we can find the optimal solution to (38) by using convex optimization tools and software such as CVX. Furthermore, we will prove that (33) without the rank constraints is tight.

Proposition 4. Without rank constraints (37), the optimal solution to problem (38) always satisfies $\operatorname{Rank}\left(\boldsymbol{Q}_{k}^{*}\right)=1, k=$ 1,2 .

Proof. Please refer to Appendix H.

Proposition 4 shows that the rank relaxation is always tight and the optimal solution to problem (38) is same as (33). Therefore, the optimal solution to the original problem (27) or (33) can be achieved by solving a convex problem. Specifically, one can first achieve the optimal $\left\{\boldsymbol{Q}_{1}^{*}, \boldsymbol{Q}_{2}^{*}\right\}$ to problem (38) by using, e.g., the tool of CVX. Then, using the SVD of $\boldsymbol{Q}_{k}, k=1,2$, one can easily obtain the optimal beamforming solution for TPM.

\section{B. Optimal beamforming in closed form with quasi-degraded channels}

In the following Proposition, the optimal beamforming vectors are obtained in closed form.

Proposition 5. The optimal solution to problem (27) is

$$
\begin{aligned}
& \boldsymbol{w}_{1}^{*}=\sqrt{\frac{A_{1}^{\min } \sigma^{2}}{\mathbf{h}_{1}^{H} \tilde{\boldsymbol{w}}_{1} \tilde{\boldsymbol{w}}_{1}{ }^{H} \mathbf{h}_{1} \tilde{\boldsymbol{w}}_{1}{ }^{H} \tilde{\boldsymbol{w}}_{1}} \tilde{\boldsymbol{w}}_{1},} \\
& \boldsymbol{w}_{2}^{*}=\sqrt{\frac{\left(A_{1}^{\min } \mathbf{h}_{2}^{H} \tilde{\boldsymbol{w}}_{1} \tilde{\boldsymbol{w}}_{1}{ }^{H} \mathbf{h}_{2}+\mathbf{h}_{1}^{H} \tilde{\boldsymbol{w}}_{1} \tilde{\boldsymbol{w}}_{1}{ }^{H} \mathbf{h}_{1}\right) A_{2}^{\min } \sigma^{2}}{\mathbf{h}_{1}^{H} \tilde{\boldsymbol{w}}_{1} \tilde{\boldsymbol{w}}_{1}{ }^{H} \mathbf{h}_{1} \mathbf{h}_{2}^{H} \mathbf{h}_{2} \mathbf{h}_{2}^{H} \mathbf{h}_{2}}},
\end{aligned}
$$

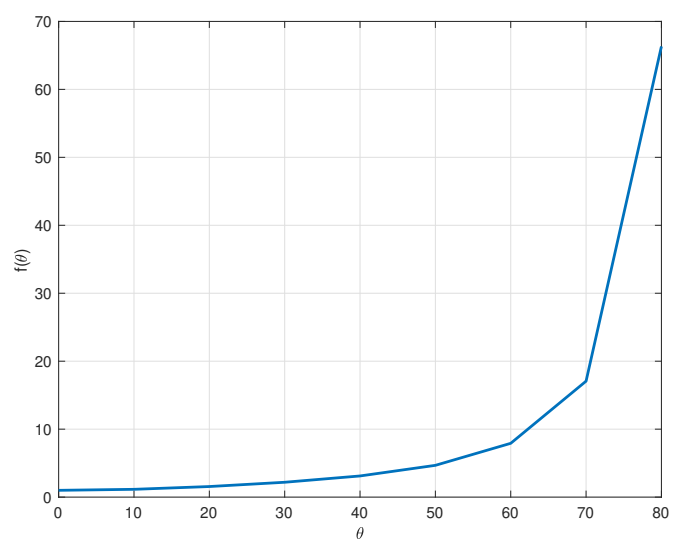

Figure 1. The function of $f(\theta)$

where

$$
\tilde{\boldsymbol{w}}_{1}=\frac{\left(\boldsymbol{I}_{T}+A_{2}^{\min } \frac{\boldsymbol{h}_{2} \boldsymbol{h}_{2}^{H}}{\boldsymbol{h}_{2}^{H} \boldsymbol{h}_{2}}\right)^{-1} \boldsymbol{h}_{1}}{\left|\left(\boldsymbol{I}_{T}+A_{2}^{\min } \frac{\boldsymbol{h}_{2} \boldsymbol{h}_{2}^{H}}{\boldsymbol{h}_{2}^{H} \boldsymbol{h}_{2}}\right)^{-1} \boldsymbol{h}_{1}\right|} .
$$

Proof. Please refer to Appendix I.

Corollary 3. The optimal beamforming proposed in (42) achieves the same performance as DPC.

Proof. Please refer to Appendix J.

In the special case with two users, since the TPM problem is equivalently transformed into a convex problem, we can achieve the global optimal beamforming, which is even characterized in closed form. In addition, according to Corollary 3 , the optimal beamforming solution achieves the same performance as DPC. Hence, according to Definition 1, the channels of users are quasi-degraded at this optimal point. However, the optimal beamforming in Proposition 5 can be achieved only with some condition of channel gains and QoS thresholds, which is described in the following.

Proposition 6. The optimal beamforming solution in (42) can be achieved if and only if

$$
\begin{array}{r}
C 3: A_{1}^{\min }+1-\frac{A_{1}^{\min } \boldsymbol{h}_{2}^{H} \boldsymbol{h}_{1} \boldsymbol{h}_{1}^{H} \boldsymbol{h}_{2} \boldsymbol{h}_{1}^{H} \boldsymbol{h}_{2} \boldsymbol{h}_{2}^{H} \boldsymbol{h}_{1}}{\left(\left(1+A_{2}^{\min }\right) \boldsymbol{h}_{1}^{H} \boldsymbol{h}_{1} \boldsymbol{h}_{2}^{H} \boldsymbol{h}_{2}-A_{2}^{\min } \boldsymbol{h}_{1}^{H} \boldsymbol{h}_{2} \boldsymbol{h}_{2}^{H} \boldsymbol{h}_{1}\right)^{2}} \\
-\frac{\boldsymbol{h}_{1}^{H} \boldsymbol{h}_{2} \boldsymbol{h}_{2}^{H} \boldsymbol{h}_{1}}{\boldsymbol{h}_{2}^{H} \boldsymbol{h}_{2} \boldsymbol{h}_{2}^{H} \boldsymbol{h}_{2}} \leq 0
\end{array}
$$

Proof. Please refer to Appendix K.

Remark 2. The same as the quasi-degradation condition proposed in Proposition 3 if the number of users is two. In the other words, in the special case with two users, the optimal closed form solution to (27) can be achieved if and only if the users' channels are quasi-degraded.

In addition, to elaborate another important insight, condition $\mathrm{C} 3$ is rewritten as

$$
\frac{\left\|\boldsymbol{h}_{1}\right\|^{2}}{\left\|\boldsymbol{h}_{2}\right\|^{2}} \geq f(\theta)
$$




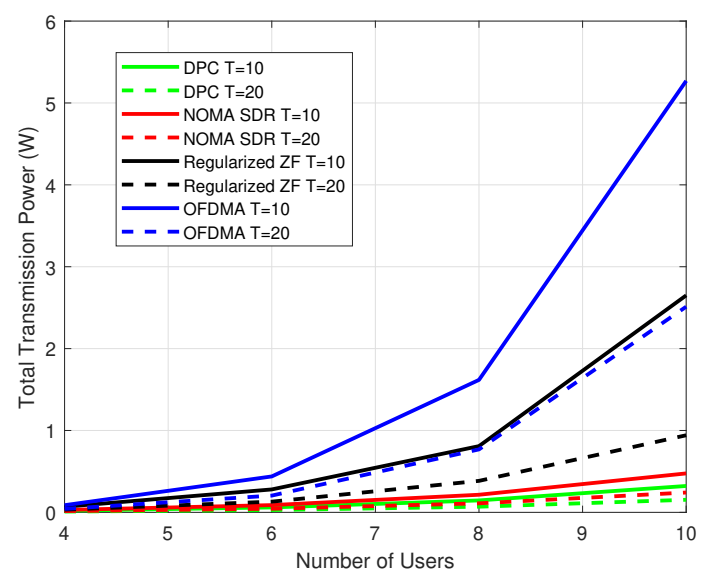

Figure 2. Total Transmission Power versus the Number of Users.

where

$$
f(\theta)=\frac{A_{1}^{\min }+1}{\cos ^{2} \theta}-\frac{A_{1}^{\min }}{\left(\left(1+A_{2}^{\min }\right) \frac{1}{\cos \theta}-A_{2}^{\min } \cos \theta\right)^{2}},
$$

and

$$
\cos ^{2} \theta=\frac{\boldsymbol{h}_{1}^{H} \boldsymbol{h}_{2} \boldsymbol{h}_{2}^{H} \boldsymbol{h}_{1}}{\boldsymbol{h}_{1}^{H} \boldsymbol{h}_{1} \boldsymbol{h}_{2}^{H} \boldsymbol{h}_{2}}
$$

and $\theta$ denotes the angle between the channels of $\mathrm{UE}_{1}$ and $\mathrm{UE}_{2}$. In Fig. 1, we show the figure of function $f(\theta)$ and it is easy to find that $f_{\min }(\theta)=f_{\min }(0)=1$. Therefore, in order to satisfy C3, we must have $\frac{\left\|\boldsymbol{h}_{1}\right\|^{2}}{\left\|\boldsymbol{h}_{2}\right\|^{2}} \geq 1$, which is consistent with our assumption. In this paper, if the channels of two users have the same direction, condition $\mathrm{C} 3$ will be satisfied, i. e., the optimal beamforming proposed in Proposition 5 can be achieved. Furthermore, if the two users do not have the same channel direction, to achieve the optimal beamforming, the channel gain between the two user should be large enough to satisfy condition $\mathrm{C} 3$.

\section{Simulation Results}

In this section, we show the performance of our proposed beamforming solution to the TPM problem in the downlink MISO NOMA systems. In our simulation, the singleantenna users are distributed uniformly within the circle and the radius of the circle is 50 meters around the BS. The channel coefficient follows an i.i.d. Gaussian distribution as $\mathbf{g}_{k} \sim \mathcal{C N}(0, \mathbf{I})$ for $k=1, \cdots, N$ and the exponent of path loss is $\alpha=3$. The noise power is $\sigma^{2}=0.0001$. In addition, for multiple-users case, the QoS threshold of user $n$ is given as $R_{n}^{\min }=(0.2+0.01(n-1)) \mathrm{bps} / \mathrm{Hz}$. For two-users case, the QoS thresholds of user 1 and user 2 are respectively set as $R_{1}^{\mathrm{min}}=1 \mathrm{bps} / \mathrm{Hz}$ and $R_{2}^{\min }=1.2 \mathrm{bps} / \mathrm{Hz}$. In the simulations, we compare the proposed beamforming design, i.e., the beamforming using SDR method and the optimal beamforming in closed form, with the OFDMA scheme and the two widely used schemes, including DPC and the regularized ZF [34].

Fig. 2 depicts the total transmission power of MISO NOMA systems versus the number of users with different numbers of antennas, i.e., $T=10$ and $T=20$. It is seen that the proposed

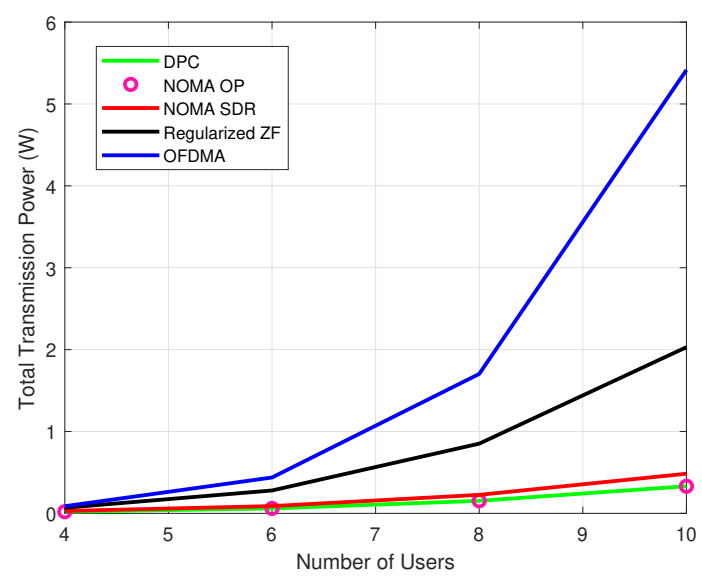

Figure 3. Total Transmission Power versus the Number of Users.

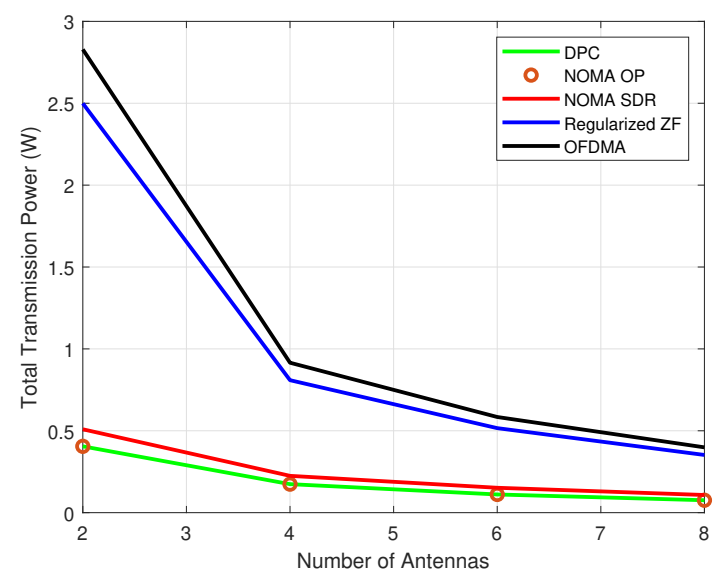

Figure 4. Total Transmission Power versus the Number of Antennas for Multiple Users.

beamforming using SDR in the downlink MISO NOMA systems slightly results in a performance loss compared to the optimal DPC scheme. Compared with ZF and OFDMA schemes, MISO NOMA beamforming yields a significant performance gain, particularly when the number of users is large and the number of antennas is small.

In Fig. 3, we focus on the situation where the channels of users are quasi-degraded. In this figure, the number of antennas is $T=10$. As expected, the optimal beamforming proposed in this paper indeed achieves better performance than the SDR method in which the SDR method solves the optimization problem by omitting the rank constraint, which induces the suboptimal solution. Furthermore, it is easy to find that our proposed optimal beamforming solution achieves the same performance as DPC, which means with quasidegradation condition, the downlink MISO NOMA scheme is able to achieve the best performance of MISO systems. In addition, there are similar phenomenons as in Fig. 2 that NOMA scheme can bring better performance than the existing ZF and OFDMA schemes, especially when the number of users is large.

Fig. 4 shows the total transmission power versus the number 


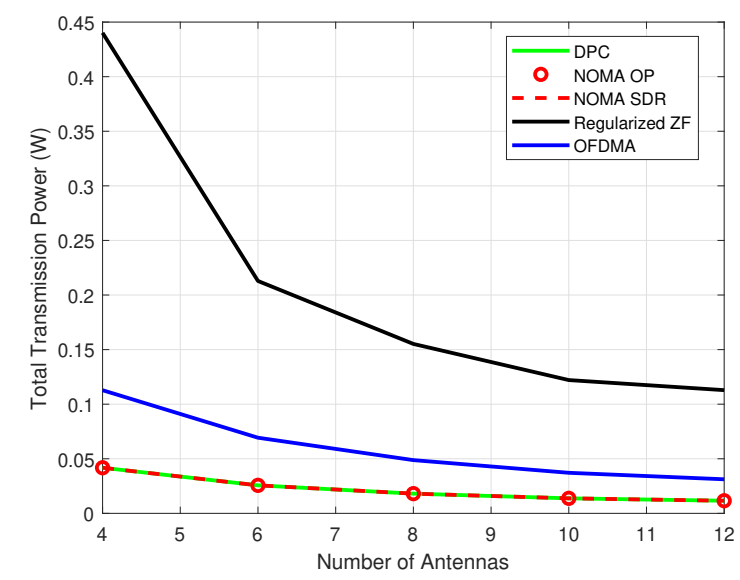

Figure 5. Total Transmission Power versus the Number of Antennas for Two Users.

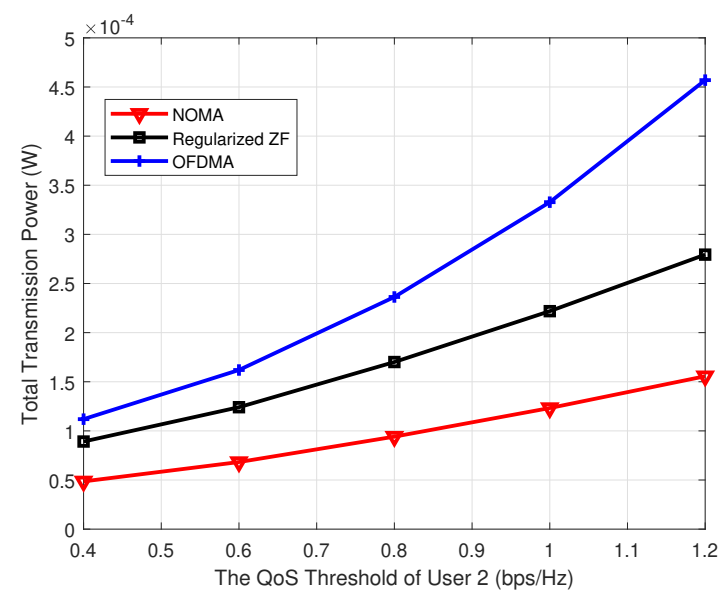

Figure 6. Total Transmission Power versus the Number of Antennas for Two Users.

of antennas. In this figure, the channels are assumed to be quasi-degraded and the number of users is $N=6$. One can observe the similar phenomenons as in Fig. 3 that NOMA outperforms OFDMA and ZF scheme, the proposed optimal beamforming lead to lower power than the SDR method and the MISO NOMA scheme can achieve the same performance as DPC. In addition, with the increasing of the number of antennas, the gap between the NOMA scheme and the conventional scheme, i.e., the OFDMA and ZF, will increase.

Fig. 5 displays the total transmission power versus the number of antennas in the special case with two users. In this figure, the channels of these two users are assumed to be quasi-degraded. Different from Fig. 3 and Fig. 4, the proposed beamforming using SDR is also optimal and achieves the same performance as the proposed optimal beamforming in closed form and the DPC scheme. This is because, in the scenario where the number of users is $N=2$, the rank relaxation is always tight. Therefore, in the special case with two users, the optimal beamforming can be obtained either using SDR method or using our proposed optimal beamforming in closed form.
In Fig. 6, we depict the impact of QoS thresholds for the two-users MISO NOMA case. In this figure, the QoS threshold of user 1 is given as $R_{1}^{\min }=1 \mathrm{bps} / \mathrm{Hz}$ while the QoS threshold of user 2 , i.e., $R_{2}^{\mathrm{min}}$, ranges from $0.4 \mathrm{bps} / \mathrm{Hz}$ to $1.2 \mathrm{bps} / \mathrm{Hz}$. As expected, NOMA always outperforms ZF and OFDMA whenever $R_{1}^{\min } \geq R_{2}^{\min }$ or $R_{1}^{\min }<R_{2}^{\mathrm{min}}$. In addition, one can easily find out that the gap between NOMA and ZF or OFDMA becomes larger when user 2's QoS threshold increases.

\section{CONCLUSION}

In this paper, in order to obtain the minimum transmission power of all users, we optimized the beamforming vectors in the downlink multi-users MISO NOMA systems. Specifically, we first provided the beamforming via SDR method. Then, we introduced the definition of quasi-degradation for multiple users and characterized the optimal beamforming in closed form with quasi-degradation condition. In addition, in the special case with two users, we showed that the original nonconvex TPM problem could be solved via transferring into an equivalent convex problem and the optimal beamforming was also characterized in closed form with quasi-degradation condition.

\section{APPENDIX}

\section{A. Proof of Lemma 1}

Let $\mathcal{C}_{N}$ and $\mathcal{C}_{D}$ respectively denote the feasible region of the optimization problem (5) and (14). It is easy to find that $\mathcal{C}_{N} \subseteq \mathcal{C}_{D}$. Therefore, the optimal solution to (5) is also a feasible solution to (14). According to the definition of quasidegradation in Definition 1, we have $P_{N O M A}=P_{D P C}$, which means $\left\{\boldsymbol{w}_{k}^{* N O M A}\right\}_{k=1}^{N}$ can achieve the optimal value of (14). Therefore, $\left\{\boldsymbol{w}_{k}^{* N O M A}\right\}_{k=1}^{N}$ is also the optimal solution to (14), i.e., $\left\{\boldsymbol{w}_{k}^{* D P C}\right\}_{k=1}^{N}=\left\{\boldsymbol{w}_{k}^{* N O M A}\right\}_{k=1}^{N}$.

\section{B. Proof of Lemma 2}

Note that, adding an arbitrary phase rotation to the beamforming vectors has no effect to the rate of $\mathrm{UE}_{k}$. In other words, if $\left\{\mathbf{w}_{k}\right\}$ is optimal, so is $\left\{\mathbf{w}_{k} e^{j \phi_{k}}\right\}$ [35], [36]. Without loss of optimality, we apply this phase ambiguity to rotate the beamforming vectors such that the inner product $\left\{\mathbf{h}_{k}^{H} \mathbf{w}_{k}\right\}_{k=1}^{N}$ are real valued and positive.

\section{Proof of Proposition 1}

Since the cost function $\sum_{k=1}^{N}\left\|\boldsymbol{w}_{k}\right\|_{2}^{2}$ in (14) is a convex function, the non-convexity lies in the QoS constraints. To find out the hidden convexity of the QoS constraints, we use a method from [8]. First, according to Lemma 2, we can add an arbitrary phase rotation to the beamforming vectors without affecting the rate of $\mathrm{UE}_{k}$, i.e., if $\left\{\mathbf{w}_{k}\right\}$ is optimal, so is $\left\{\mathbf{w}_{k} e^{j \phi_{k}}\right\}$. Without loss of optimality, we rotate the phase such that the inner product $\mathbf{h}_{k}^{H} \mathbf{w}_{k}$ is real valued and positive, implying $\sqrt{\left|\mathbf{h}_{k}^{H} \mathbf{w}_{k}\right|^{2}}=\mathbf{h}_{k}^{H} \mathbf{w}_{k}$ for $k=1, \cdots, N$.

Hence, we may take square root of the constraints (16) in (14) and then the constraints become second-order cone 
programming constraints [8], [35], [36], which is in fact convex. Therefore, the TPM problem can be equivalently rewritten as (18), which is convex.

\section{Proof of Corollary 1}

The Lagrangian of problem (18) is given by

$$
\begin{aligned}
L= & \sum_{k=1}^{N}\left\|\boldsymbol{w}_{k}\right\|_{2}^{2} \\
& +\mu_{k}\left(\sqrt{\sum_{j=1}^{k-1} 1 / \sigma^{2} \mathbf{h}_{k}^{H} \boldsymbol{w}_{j} \boldsymbol{w}_{j}^{H} \mathbf{h}_{k}+1}-\frac{1}{\sigma \sqrt{A_{k}^{\min }}} \mathbf{h}_{k}^{H} \boldsymbol{w}_{k}\right),
\end{aligned}
$$

where $\mu_{k}$ for $k=1, \cdots, N$ are the Lagrangian multipliers. Let

$$
t_{k}=\sqrt{\sum_{j=1}^{k-1} 1 / \sigma^{2} \mathbf{h}_{k}^{H} \boldsymbol{w}_{j} \boldsymbol{w}_{j}^{H} \mathbf{h}_{k}+1}+\frac{1}{\sigma \sqrt{A_{k}^{\min }}} \mathbf{h}_{k}^{H} \boldsymbol{w}_{k},
$$

then (48) can be rewritten as

$$
\begin{aligned}
L & =\sum_{k=1}^{N}\left\|\boldsymbol{w}_{k}\right\|_{2}^{2} \\
& +\frac{\mu_{k}}{t_{k}}\left(\sum_{j=1}^{k-1} 1 / \sigma^{2} \mathbf{h}_{k}^{H} \boldsymbol{w}_{j} \boldsymbol{w}_{j}^{H} \mathbf{h}_{k}+1-\frac{1}{\sigma^{2} A_{k}^{\min }} \boldsymbol{h}_{k}^{H} \boldsymbol{w}_{k} \boldsymbol{w}_{k}^{H} \boldsymbol{h}_{k}\right) .
\end{aligned}
$$

Note that $t_{k}$ for $k=1, \cdots, N$ are strictly positive, hence we can change the optimization variable to $\lambda_{k}=\mu_{k} / t_{k}, k=$ $1, \cdots, N$. Under this change of variables, the Lagrangian of the convex form of problem (18) is then exactly the same as the Lagrangian of the original form of problem (14).

\section{E. Proof of Proposition 2}

The Lagrangian of (14) is given by

$$
\begin{aligned}
L & =\sum_{k=1}^{N}\left\|\boldsymbol{w}_{k}\right\|_{2}^{2} \\
& +\lambda_{k}\left(\sum_{j=1}^{k-1} \frac{1}{\sigma^{2}} \mathbf{h}_{k}^{H} \boldsymbol{w}_{j} \boldsymbol{w}_{j}^{H} \mathbf{h}_{k}+1-\frac{1}{\sigma^{2} A_{k}^{\min }} \boldsymbol{h}_{k}^{H} \boldsymbol{w}_{k} \boldsymbol{w}_{k}^{H} \boldsymbol{h}_{k}\right),
\end{aligned}
$$

where $\lambda_{k} \geq 0$ for $k=1, \cdots, N$ are the Lagrangian multipliers. The corresponding Karush-Kuhn-Tucker (KKT) conditions are:

$$
\begin{gathered}
\frac{\partial L}{\partial \boldsymbol{w}_{k}}=\boldsymbol{w}_{k}+\sum_{j=k+1}^{N} \frac{\lambda_{j}}{\sigma^{2}} \mathbf{h}_{j} \mathbf{h}_{j}^{H} \boldsymbol{w}_{k}-\frac{\lambda_{k}}{\sigma^{2} A_{k}^{\min }} \mathbf{h}_{k} \mathbf{h}_{k}^{H} \boldsymbol{w}_{k}=0 \\
\lambda_{k}\left(\sum_{j=1}^{k-1} \frac{1}{\sigma^{2}} \mathbf{h}_{k}^{H} \boldsymbol{w}_{j} \boldsymbol{w}_{j}^{H} \mathbf{h}_{k}+1-\frac{1}{\sigma^{2} A_{k}^{\min }} \boldsymbol{h}_{k}^{H} \boldsymbol{w}_{k} \boldsymbol{w}_{k}^{H} \boldsymbol{h}_{k}\right)=0 .
\end{gathered}
$$

From (52), we obtain,

$$
\boldsymbol{w}_{k}=\left(\boldsymbol{I}_{T}+\sum_{j=k+1}^{N} \frac{\lambda_{j}}{\sigma^{2}} \mathbf{h}_{j} \mathbf{h}_{j}^{H}\right)^{-1} \mathbf{h}_{k} \frac{\lambda_{k}}{\sigma^{2} A_{k}^{\min }} \mathbf{h}_{k}^{H} \boldsymbol{w}_{k} .
$$

Here, according to Lemma $2, \frac{\lambda_{k}}{\sigma^{2} A_{k}^{\min }} \mathbf{h}_{k}^{H} \boldsymbol{w}_{k}$ can be a scalar value and hence the optimal beamforming for $\mathrm{UE}_{k}$ is parallel to $\left(\boldsymbol{I}_{T}+\sum_{j=k+1}^{N} \frac{\lambda_{j}}{\sigma^{2}} \mathbf{h}_{j} \mathbf{h}_{j}^{H}\right)^{-1} \mathbf{h}_{k}$, i.e., the optimal beamforming for $\mathrm{UE}_{k}$ can be given as

$$
\boldsymbol{w}_{k}=\sqrt{p_{k}} \tilde{\boldsymbol{w}}_{k},
$$

where $\tilde{\boldsymbol{w}}_{k}$ denotes the uniformed beamforming direction for $\mathrm{UE}_{k}$ and given as

$$
\tilde{\boldsymbol{w}}_{k}=\frac{\left(\boldsymbol{I}_{T}+\sum_{j=k+1}^{N} \frac{\lambda_{j}}{\sigma^{2}} \mathbf{h}_{j} \mathbf{h}_{j}^{H}\right)^{-1} \mathbf{h}_{k}}{\left|\left(\boldsymbol{I}_{T}+\sum_{j=k+1}^{N} \frac{\lambda_{j}}{\sigma^{2}} \mathbf{h}_{j} \mathbf{h}_{j}^{H}\right)^{-1} \mathbf{h}_{k}\right|} .
$$

Here, the Lagrangian multipliers $\lambda_{k}$ for $k=1, \cdots, N$ is obtained from (54), i. e.,

$$
\left(\boldsymbol{I}_{T}+\sum_{j=k+1}^{N} \frac{\lambda_{j}}{\sigma^{2}} \mathbf{h}_{j} \mathbf{h}_{j}^{H}\right)^{-1} \mathbf{h}_{k} \frac{\lambda_{k}}{\sigma^{2} A_{k}^{\min }} \mathbf{h}_{k}^{H}=\boldsymbol{I}_{T} .
$$

The Lagrangian multipliers $\lambda_{k}$ for $k=1, \cdots, N$ is then given as in (21). In addition, in (55), $p_{k}$ denotes the optimal power allocated to $\mathrm{UE}_{k}$, which can be obtained from (53) with $\lambda_{k}>$ 0 , i.e.,

$$
\sum_{j=1}^{k-1} \frac{1}{\sigma^{2}} \mathbf{h}_{k}^{H} \boldsymbol{w}_{j} \boldsymbol{w}_{j}^{H} \mathbf{h}_{k}+1-\frac{1}{\sigma^{2} A_{k}^{\min }} \boldsymbol{h}_{k}^{H} \boldsymbol{w}_{k} \boldsymbol{w}_{k}^{H} \boldsymbol{h}_{k}=0,
$$

implying

$$
p_{1} \mathbf{h}_{1}^{H} \tilde{\boldsymbol{w}}_{1} \tilde{\boldsymbol{w}}_{1}^{H} \mathbf{h}_{1}-A_{1}^{\min } \sigma^{2}=0
$$

$$
A_{k}^{\min }\left(\sum_{j=1}^{k-1} p_{j} \mathbf{h}_{k}^{H} \tilde{\boldsymbol{w}}_{j} \tilde{\boldsymbol{w}}_{j}{ }^{H} \mathbf{h}_{k}+\sigma^{2}\right)-p_{k} \mathbf{h}_{k}^{H} \tilde{\boldsymbol{w}}_{k} \tilde{\boldsymbol{w}}_{k}{ }^{H} \mathbf{h}_{k}=0,
$$

$$
k=2, \cdots, N .
$$

Therefore the optimal powers are obtained as in (23).

\section{F. Proof of Corollary 2}

According to Proposition 2, the optimal transmit powers are given in (23), hence, for $k=2, \cdots, N$, we have

$$
p_{k} \mathbf{h}_{k}^{H} \tilde{\boldsymbol{w}}_{k} \tilde{\boldsymbol{w}}_{k}{ }^{H} \mathbf{h}_{k}=A_{k}^{\min } \mathbf{h}_{k}^{H}\left(\sum_{j=1}^{k-1} p_{j} \tilde{\boldsymbol{w}}_{j} \tilde{\boldsymbol{w}}_{j}{ }^{H}\right) \mathbf{h}_{k}+A_{k}^{\min } \sigma^{2},
$$

therefore

$$
p_{k} \tilde{\boldsymbol{w}}_{k} \tilde{\boldsymbol{w}}_{k}{ }^{H}-A_{k}^{\min } \sum_{j=1}^{k-1} p_{j} \tilde{\boldsymbol{w}}_{j} \tilde{\boldsymbol{w}}_{j}{ }^{H}=\frac{A_{k}^{\min } \sigma^{2}}{\mathbf{h}_{k}^{H} \mathbf{h}_{k}} \boldsymbol{I}_{T} .
$$

Since $\tilde{\boldsymbol{w}}_{k}$, for $k=1, \cdots, N$, are normalized vectors, i.e., $\operatorname{Tr}\left(\tilde{\boldsymbol{w}}_{k} \tilde{\boldsymbol{w}}_{k}{ }^{H}\right)=\tilde{\boldsymbol{w}}_{k}{ }^{H} \tilde{\boldsymbol{w}}_{k}=1$, then 


$$
\frac{p_{k}}{A_{k}^{\min }}-\sum_{j=1}^{k-1} p_{j}=\frac{\sigma^{2}}{\mathbf{h}_{k}^{H} \mathbf{h}_{k}},
$$

which implies

$$
\frac{p_{k+1}}{A_{k+1}^{\min }}-\sum_{j=1}^{k} p_{j}=\frac{\sigma^{2}}{\mathbf{h}_{k+1}^{H} \mathbf{h}_{k+1}} .
$$

Subtracting (63) from (64) yields

$$
\frac{1}{A_{k+1}^{\min }}\left(p_{k+1}+B\right)=\left(\frac{1}{A_{k}^{\min }}+1\right)\left(p_{k}+B\right),
$$

where

$$
B=\frac{\sigma^{2}\left(\mathbf{h}_{k}^{H} \mathbf{h}_{k}-\mathbf{h}_{k+1}^{H} \mathbf{h}_{k+1}\right) A_{k}^{\min } A_{k+1}^{\min }}{\mathbf{h}_{k+1}^{H} \mathbf{h}_{k+1} \mathbf{h}_{k}^{H} \mathbf{h}_{k}\left(A_{k+1}^{\min } A_{k}^{\min }+A_{k}^{\min }+A_{k+1}^{\min }\right)}>0 .
$$

Given condition $\mathrm{C} 1$ is satisfied, one can write

$$
\frac{p_{k+1}+B}{p_{k}+B}=\frac{A_{k}^{\min } A_{k+1}^{\min }+A_{k+1}^{\min }}{A_{k}^{\min }} \geq 1 .
$$

Since $B>0$, we always have $p_{k+1}>p_{k}$ as in condition $\mathrm{C}$.

\section{G. Proof of Proposition 3}

First, we prove the sufficiency of the condition. According to Proposition 2, we provides the optimal solution to (14). Here, if condition $\mathrm{C} 2$ is satisfied, one can easily find that the optimal solution, $\boldsymbol{w}_{k}^{*}$, for $k=1, \cdots, N$, in Proposition 2 is also a solution to problem (5). Therefore, according to Definition 1, the broadcast channels, $\left\{\mathbf{h}_{k}\right\}_{k=1}^{N}$, are quasidegraded.

To prove the necessity of the condition, if the broadcast channels $\left\{\mathbf{h}_{k}\right\}_{k=1}^{N}$ are quasi-degraded with respect to $\left\{A_{k}^{\min }\right\}_{k=1}^{N}$, according to Definition 1 and Proposition 2, the proposed beamforming is the optimal solution to (5). Therefore, the proposed beamforming should satisfy the constraints in (5), which then implies condition $\mathrm{C} 2$ and completes the proof.

\section{H. Proof of Proposition 4}

It is shown in [37] that there exists an optimal solution $\left\{\boldsymbol{Q}_{1}^{*}, \boldsymbol{Q}_{2}^{*}\right\}$ of the SDR problem (38) such that

$$
\operatorname{rank}^{2}\left(\boldsymbol{Q}_{1}^{*}\right)+\operatorname{rank}^{2}\left(\boldsymbol{Q}_{2}^{*}\right) \leq 3 .
$$

We also note that, in order to meet the positive QoS constraints, $\boldsymbol{Q}_{k}^{*} \neq \mathbf{0}$, i.e., $\operatorname{rank}\left(\boldsymbol{Q}_{k}^{*}\right) \geq 1, k=1,2$. Therefore, there must exist an optimal solution such that $\operatorname{rank}\left(\boldsymbol{Q}_{1}^{*}\right)=$ $\operatorname{rank}\left(\boldsymbol{Q}_{2}^{*}\right)=1$.

\section{Proof of Proposition 5}

The Lagrangian of the convex problem (38) is

$$
\begin{aligned}
L= & \operatorname{Tr}\left(\boldsymbol{Q}_{1}+\boldsymbol{Q}_{2}\right)+\lambda_{1}\left(1-\frac{1}{\sigma^{2} A_{1}^{\min }} \mathbf{h}_{1}^{H} \boldsymbol{Q}_{1} \mathbf{h}_{1}\right) \\
& +\lambda_{2}\left(\frac{1}{\sigma^{2}} \mathbf{h}_{1}^{H} \boldsymbol{Q}_{1} \mathbf{h}_{1}-\frac{1}{\sigma^{2} A_{2}^{\min }} \mathbf{h}_{1}^{H} \boldsymbol{Q}_{2} \mathbf{h}_{1}+1\right) \\
& +\lambda_{3}\left(\frac{1}{\sigma^{2}} \mathbf{h}_{2}^{H} \boldsymbol{Q}_{1} \mathbf{h}_{2}-\frac{1}{\sigma^{2} A_{2}^{\min }} \mathbf{h}_{2}^{H} \boldsymbol{Q}_{2} \mathbf{h}_{2}+1\right) \\
& -\operatorname{Tr}\left(\mathbf{\Upsilon}_{1} \boldsymbol{Q}_{1}+\mathbf{\Upsilon}_{2} \boldsymbol{Q}_{2}\right),
\end{aligned}
$$

where $\lambda_{l} \geq 0, l=1, \ldots, 3$, and $\Upsilon_{k} \succeq \mathbf{0}, k=1,2$, are the Lagrangian multipliers. Applying KKT conditions of (38) yields

$$
\begin{gathered}
\frac{\partial L}{\partial \boldsymbol{Q}_{1}}=I-\lambda_{1} \mathbf{h}_{1} \mathbf{h}_{1}^{H}+\frac{\lambda_{2}}{\sigma^{2}} \mathbf{h}_{1} \mathbf{h}_{1}^{H}+\frac{\lambda_{3}}{\sigma^{2}} \mathbf{h}_{2} \mathbf{h}_{2}^{H}-\mathbf{\Upsilon}_{1}=\mathbf{0} \\
\frac{\partial L}{\partial \boldsymbol{Q}_{2}}=I-\frac{\lambda_{2}}{\sigma^{2} A_{2}^{\min }} \mathbf{h}_{1} \mathbf{h}_{1}^{H}-\frac{\lambda_{3}}{\sigma^{2} A_{2}^{\min }} \mathbf{h}_{2} \mathbf{h}_{2}^{H}-\mathbf{\Upsilon}_{2}=\mathbf{0} \\
\lambda_{1}\left(1-\frac{1}{\sigma^{2} A_{1}^{\min }} \mathbf{h}_{1}^{H} \boldsymbol{Q}_{1} \mathbf{h}_{1}\right)=0 \\
\lambda_{2}\left(\frac{1}{\sigma^{2}} \mathbf{h}_{1}^{H} \boldsymbol{Q}_{1} \mathbf{h}_{1}-\frac{1}{\sigma^{2} A_{2}^{\min }} \mathbf{h}_{1}^{H} \boldsymbol{Q}_{2} \mathbf{h}_{1}+1\right)=0 \\
\lambda_{3}\left(\frac{1}{\sigma^{2}} \mathbf{h}_{2}^{H} \boldsymbol{Q}_{1} \mathbf{h}_{2}-\frac{1}{\sigma^{2} A_{2}^{\min }} \mathbf{h}_{2}^{H} \boldsymbol{Q}_{2} \mathbf{h}_{2}+1\right)=0 \\
\mathbf{\Upsilon}_{k} \boldsymbol{Q}_{k}=\mathbf{0}, k=1,2
\end{gathered}
$$

Multiplying both sides of equation (70), and (71), by $\boldsymbol{Q}_{1}$, and $\boldsymbol{Q}_{2}$, respectively, we obtain

$$
\begin{gathered}
\boldsymbol{Q}_{1}-\lambda_{1} \mathbf{h}_{1} \mathbf{h}_{1}^{H} \boldsymbol{Q}_{1}+\frac{\lambda_{2}}{\sigma^{2}} \mathbf{h}_{1} \mathbf{h}_{1}^{H} \boldsymbol{Q}_{1}+\frac{\lambda_{3}}{\sigma^{2}} \mathbf{h}_{2} \mathbf{h}_{2}^{H} \boldsymbol{Q}_{1}=0 \\
\boldsymbol{Q}_{2}-\frac{\lambda_{2}}{\sigma^{2} A_{2}^{\min }} \mathbf{h}_{1} \mathbf{h}_{1}^{H} \boldsymbol{Q}_{2}-\frac{\lambda_{3}}{\sigma^{2} A_{2}^{\min }} \mathbf{h}_{2} \mathbf{h}_{2}^{H} \boldsymbol{Q}_{2}=0
\end{gathered}
$$

Since the SDR problem in (38) is always tight, it is easy to have $\boldsymbol{Q}_{k}=\boldsymbol{w}_{k} \boldsymbol{w}_{k}^{H}, k=1,2$. Therefore, (76), (77), and the KKT conditions, i.e., (72) to (74), are reduced to

$\boldsymbol{w}_{1} \boldsymbol{w}_{1}^{H}-\lambda_{1} \mathbf{h}_{1} \mathbf{h}_{1}^{H} \boldsymbol{w}_{1} \boldsymbol{w}_{1}^{H} \frac{\lambda_{2}}{\sigma^{2}} \mathbf{h}_{1} \mathbf{h}_{1}^{H} \boldsymbol{w}_{1} \boldsymbol{w}_{1}^{H} \frac{\lambda_{3}}{\sigma^{2}} \mathbf{h}_{2} \mathbf{h}_{2}^{H} \boldsymbol{w}_{1} \boldsymbol{w}_{1}^{H}=\mathbf{0}$,

$\boldsymbol{w}_{2} \boldsymbol{w}_{2}^{H}-\frac{\lambda_{2}}{\sigma^{2} A_{2}^{\min }} \mathbf{h}_{1} \mathbf{h}_{1}^{H} \boldsymbol{w}_{2} \boldsymbol{w}_{2}^{H}-\frac{\lambda_{3}}{\sigma^{2} A_{2}^{\min }} \mathbf{h}_{2} \mathbf{h}_{2}^{H} \boldsymbol{w}_{2} \boldsymbol{w}_{2}^{H}=\mathbf{0}$,

$$
\begin{gathered}
\lambda_{1}\left(A_{1}^{\min } \sigma^{2}-\mathbf{h}_{1}^{H} \boldsymbol{w}_{1} \boldsymbol{w}_{1}^{H} \mathbf{h}_{1}\right)=0 \\
\lambda_{2}\left(\frac{1}{\sigma^{2}} \mathbf{h}_{1}^{H} \boldsymbol{w}_{1} \boldsymbol{w}_{1}^{H} \mathbf{h}_{1}+1-\frac{1}{\sigma^{2} A_{2}^{\min }} \mathbf{h}_{1}^{H} \boldsymbol{w}_{2} \boldsymbol{w}_{2}^{H} \mathbf{h}_{1}\right)=0 \\
\lambda_{3}\left(\frac{1}{\sigma^{2}} \mathbf{h}_{2}^{H} \boldsymbol{w}_{1} \boldsymbol{w}_{1}^{H} \mathbf{h}_{2}+1-\frac{1}{\sigma^{2} A_{2}^{\min }} \mathbf{h}_{2}^{H} \boldsymbol{w}_{2} \boldsymbol{w}_{2}^{H} \mathbf{h}_{2}\right)=0
\end{gathered}
$$


Furthermore, from (78) and (79), with $\boldsymbol{w}_{k} \neq \mathbf{0}$ for $k=1,2$, we have

$$
\begin{gathered}
\boldsymbol{w}_{1}-\lambda_{1} \mathbf{h}_{1} \mathbf{h}_{1}^{H} \boldsymbol{w}_{1}+\frac{\lambda_{2}}{\sigma^{2}} \mathbf{h}_{1} \mathbf{h}_{1}^{H} \boldsymbol{w}_{1}+\frac{\lambda_{3}}{\sigma^{2}} \mathbf{h}_{2} \mathbf{h}_{2}^{H} \boldsymbol{w}_{1}=\mathbf{0}, \\
\boldsymbol{w}_{2}-\frac{\lambda_{2}}{\sigma^{2} A_{2}^{\min }} \mathbf{h}_{1} \mathbf{h}_{1}^{H} \boldsymbol{w}_{2}-\frac{\lambda_{3}}{\sigma^{2} A_{2}^{\min }} \mathbf{h}_{2} \mathbf{h}_{2}^{H} \boldsymbol{w}_{2}=\mathbf{0} .
\end{gathered}
$$

It is also seen in (83) and (84) that $\lambda_{1}>0$ and $\lambda_{2}=0$, $\lambda_{3}=0$ are not possible. Therefore, three cases are possible, i.e., $\lambda_{1}>0, \lambda_{2}>0, \lambda_{3}>0 ; \lambda_{1}>0, \lambda_{2}=0, \lambda_{3}>0$; and $\lambda_{1}>0, \lambda_{2}>0, \lambda_{3}=0$.

1) The case of $\lambda_{1}>0, \lambda_{2}>0, \lambda_{3}>0$ : Using (81) and (82), we have

$$
\begin{gathered}
\frac{1}{\sigma^{2}} \mathbf{h}_{1}^{H} \boldsymbol{w}_{1} \boldsymbol{w}_{1}^{H} \mathbf{h}_{1}+1-\frac{1}{\sigma^{2} A_{2}^{\min }} \mathbf{h}_{1}^{H} \boldsymbol{w}_{2} \boldsymbol{w}_{2}^{H} \mathbf{h}_{1}=0, \\
\frac{1}{\sigma^{2}} \mathbf{h}_{2}^{H} \boldsymbol{w}_{1} \boldsymbol{w}_{1}^{H} \mathbf{h}_{2}+1-\frac{1}{\sigma^{2} A_{2}^{\min }} \mathbf{h}_{2}^{H} \boldsymbol{w}_{2} \boldsymbol{w}_{2}^{H} \mathbf{h}_{2}=0 .
\end{gathered}
$$

However, with $\left\|\mathbf{h}_{1}\right\|_{2}>\left\|\mathbf{h}_{2}\right\|_{2}$, (85) and (86) can not be satisfied simultaneously. This is because, by subtracting (86) from (85), we have

$$
\begin{aligned}
& \frac{1}{\sigma^{2}} \mathbf{h}_{1}^{H} \boldsymbol{w}_{1} \boldsymbol{w}_{1}^{H} \mathbf{h}_{1}-\frac{1}{\sigma^{2} A_{2}^{\min }} \mathbf{h}_{1}^{H} \boldsymbol{w}_{2} \boldsymbol{w}_{2}^{H} \mathbf{h}_{1} \\
& -\left(\frac{1}{\sigma^{2}} \mathbf{h}_{2}^{H} \boldsymbol{w}_{1} \boldsymbol{w}_{1}^{H} \mathbf{h}_{2}+1-\frac{1}{\sigma^{2} A_{2}^{\min }} \mathbf{h}_{2}^{H} \boldsymbol{w}_{2} \boldsymbol{w}_{2}^{H} \mathbf{h}_{2}\right) \\
= & \frac{1}{\sigma^{2}} \mathbf{h}_{1}^{H}\left(\boldsymbol{w}_{1} \boldsymbol{w}_{1}^{H}-\frac{1}{A_{2}^{\min }} \boldsymbol{w}_{2} \boldsymbol{w}_{2}^{H}\right) \mathbf{h}_{1} \\
& -\frac{1}{\sigma^{2}} \mathbf{h}_{2}^{H}\left(\boldsymbol{w}_{1} \boldsymbol{w}_{1}^{H}-\frac{1}{A_{2}^{\min }} \boldsymbol{w}_{2} \boldsymbol{w}_{2}^{H}\right) \mathbf{h}_{2} \\
= & \frac{1}{\sigma^{2}} \operatorname{Tr}\left(\left(\boldsymbol{w}_{1} \boldsymbol{w}_{1}^{H}-\frac{1}{A_{2}^{\min }} \boldsymbol{w}_{2} \boldsymbol{w}_{2}^{H}\right)\left(\mathbf{h}_{1} \mathbf{h}_{1}^{H}-\mathbf{h}_{2} \mathbf{h}_{2}^{H}\right)\right) .
\end{aligned}
$$

Since $\left\|\mathbf{h}_{1}\right\|_{2}>\left\|\mathbf{h}_{2}\right\|_{2}$, we have $\mathbf{h}_{1} \mathbf{h}_{1}^{H}-\mathbf{h}_{2} \mathbf{h}_{2}^{H} \neq \mathbf{0}$. In addition, it follows from (85) or (86) that $\boldsymbol{w}_{1} \boldsymbol{w}_{1}^{H}-\frac{1}{A_{2}^{\min }} \boldsymbol{w}_{2} \boldsymbol{w}_{2}^{H} \neq$ 0. Hence, (87) can not be equal to zero, i.e., the KKT conditions (81) and (82) can not be satisfied simultaneously. Therefore, the case $\lambda_{1}>0, \lambda_{2}>0, \lambda_{3}>0$ is not possible.

2) The case of $\lambda_{1}>0, \lambda_{2}>0, \lambda_{3}=0$ : Using (83) and (84), we have

$$
\begin{gathered}
\boldsymbol{w}_{1}-\lambda_{1} \boldsymbol{h}_{1} \boldsymbol{h}_{1}^{H} \boldsymbol{w}_{1}+\frac{\lambda_{2}}{\sigma^{2}} \boldsymbol{h}_{1} \boldsymbol{h}_{1}^{H} \boldsymbol{w}_{1}=0 \\
\boldsymbol{w}_{2}-\frac{\lambda_{2}}{\sigma^{2} A_{2}^{\min }} \boldsymbol{h}_{1} \boldsymbol{h}_{1}^{H} \boldsymbol{w}_{2}=0
\end{gathered}
$$

therefore

$$
\begin{aligned}
& \boldsymbol{w}_{1}=\left(\boldsymbol{I}_{T}+\frac{\lambda_{2}}{\sigma^{2}} \boldsymbol{h}_{1} \boldsymbol{h}_{1}^{H}\right)^{-1} \boldsymbol{h}_{1} \lambda_{1} \boldsymbol{h}_{1}^{H} \boldsymbol{w}_{1}, \\
& \boldsymbol{w}_{2}=\boldsymbol{h}_{1} \frac{\lambda_{2}}{\sigma^{2} A_{2}^{\min }} \boldsymbol{h}_{1}^{H} \boldsymbol{w}_{2} .
\end{aligned}
$$

Here, according to Lemma $2, \lambda_{1} \boldsymbol{h}_{1}^{H} \boldsymbol{w}_{1}$ and $\frac{\lambda_{2}}{\sigma^{2} A_{2}^{\min }} \boldsymbol{h}_{1}^{H} \boldsymbol{w}_{2}$ can be both scalars. Hence, (90) and (91) show that the optimal $\boldsymbol{w}_{1}$ and $\boldsymbol{w}_{2}$ must be respectively parallel to
$\left(\boldsymbol{I}_{T}+\frac{\lambda_{2}}{\sigma^{2}} \boldsymbol{h}_{1} \boldsymbol{h}_{1}^{H}\right)^{-1} \boldsymbol{h}_{1}$, and $\boldsymbol{h}_{1}$. In the other words, the optimal beamforming vectors are $\boldsymbol{w}_{1}=\sqrt{p_{1}} \tilde{\boldsymbol{w}}_{1}, \boldsymbol{w}_{2}=\sqrt{p_{2}} \tilde{\boldsymbol{w}}_{2}$, where

$$
\begin{aligned}
& \tilde{\boldsymbol{w}}_{1}=\frac{\left(\boldsymbol{I}_{T}+\frac{\lambda_{2}}{\sigma^{2}} \boldsymbol{h}_{1} \boldsymbol{h}_{1}^{H}\right)^{-1} \boldsymbol{h}_{1}}{\left|\left(\boldsymbol{I}_{T}+\frac{\lambda_{2}}{\sigma^{2}} \boldsymbol{h}_{1} \boldsymbol{h}_{1}^{H}\right)^{-1} \boldsymbol{h}_{1}\right|}, \\
& \tilde{\boldsymbol{w}}_{2}=\frac{\boldsymbol{h}_{1}}{\left|\boldsymbol{h}_{1}\right|},
\end{aligned}
$$

denote the normalized beamforming directions and from (89), the Lagrangian multiplier is given as $\lambda_{2}=\sigma^{2} A_{2}^{\min } / \boldsymbol{h}_{1}^{H} \boldsymbol{h}_{1}$. The unknown optimal powers are then obtained by using (80) and (81) with $\lambda_{1}>0, \lambda_{2}>0$, i.e.,

$$
A_{1}^{\min } \sigma^{2}-p_{1} \boldsymbol{h}_{1}^{H} \tilde{\boldsymbol{w}}_{1} \tilde{\boldsymbol{w}}_{1}^{H} \boldsymbol{h}_{1}=0
$$

$$
\frac{1}{\sigma^{2}} p_{1} \boldsymbol{h}_{1}^{H} \tilde{\boldsymbol{w}_{1}} \widetilde{\boldsymbol{w}_{1}^{H}} \boldsymbol{h}_{1}+1-\frac{1}{\sigma^{2} A_{2}^{\min }} p_{2} \boldsymbol{h}_{1}^{H} \tilde{\boldsymbol{w}_{2}} \widetilde{\boldsymbol{w}_{2}^{H}} \boldsymbol{h}_{1}=0 .
$$

Hence, the optimal powers can be given as

$$
\begin{aligned}
& p_{1}=\frac{A_{1}^{\min } \sigma^{2}}{\boldsymbol{h}_{1}^{H} \tilde{\boldsymbol{w}}_{1} \tilde{\boldsymbol{w}}_{1}^{H} \boldsymbol{h}_{1}}, \\
& p_{2}=\frac{A_{2}^{\min } A_{1}^{\min } \sigma^{2}+A_{2}^{\min } \sigma^{2}}{\boldsymbol{h}_{1}^{H} \tilde{\boldsymbol{w}}_{2} \tilde{\boldsymbol{w}}_{2}^{H} \boldsymbol{h}_{1}} .
\end{aligned}
$$

However, in this case, i.e., $\lambda_{1}>0, \lambda_{2}>0, \lambda_{3}=0$, the corresponding optimal beamforming solution fails to hold the constraint (36) in problem (33). Specifically, the normalized beamforming direction for $\mathrm{UE}_{1}$ is

$$
\tilde{\boldsymbol{w}}_{1}=\frac{\left(\boldsymbol{I}_{T}+A_{2}^{\min } \boldsymbol{h}_{1} \boldsymbol{h}_{1}^{H} / \boldsymbol{h}_{1}^{H} \boldsymbol{h}_{1}\right)^{-1} \boldsymbol{h}_{1}}{\left|\left(\boldsymbol{I}_{T}+A_{2}^{\min } \boldsymbol{h}_{1} \boldsymbol{h}_{1}^{H} / \boldsymbol{h}_{1}^{H} \boldsymbol{h}_{1}\right)^{-1} \boldsymbol{h}_{1}\right|}=\frac{\boldsymbol{\Lambda} \boldsymbol{h}_{1}}{\left|\boldsymbol{\Lambda} \boldsymbol{h}_{1}\right|} .
$$

Using the Sherman-Morrison equation, we have

$$
\begin{aligned}
\boldsymbol{\Lambda} & =\left(\boldsymbol{I}_{T}+A_{2}^{\min } \boldsymbol{h}_{1} \boldsymbol{h}_{1}^{H} / \boldsymbol{h}_{1}^{H} \boldsymbol{h}_{1}\right)^{-1} \\
& =\boldsymbol{I}_{T}-\frac{A_{2}^{\min } \boldsymbol{h}_{1} \boldsymbol{h}_{1}^{H} / \boldsymbol{h}_{1}^{H} \boldsymbol{h}_{1}}{1+A_{2}^{\min }} .
\end{aligned}
$$

Therefore,

$$
\begin{aligned}
& \frac{1}{\sigma^{2}} \boldsymbol{h}_{2}^{H} \boldsymbol{w}_{1} \boldsymbol{w}_{1}^{H} \boldsymbol{h}_{2}+1-\frac{1}{\sigma^{2} A_{2}^{\min }} \boldsymbol{h}_{2}^{H} \boldsymbol{w}_{2} \boldsymbol{w}_{2}^{H} \boldsymbol{h}_{2} \\
= & \frac{p_{1}}{\sigma^{2}} \boldsymbol{h}_{2}^{H} \tilde{\boldsymbol{w}}_{1} \tilde{\boldsymbol{w}}_{1}^{H} \boldsymbol{h}_{2}-\frac{p_{2}}{\sigma^{2} A_{2}^{\min }} \boldsymbol{h}_{2}^{H} \tilde{\boldsymbol{w}}_{2} \tilde{\boldsymbol{w}}_{2}^{H} \boldsymbol{h}_{2}+1 \\
= & \frac{A_{1}^{\min } \boldsymbol{h}_{2}^{H} \tilde{\boldsymbol{w}}_{1} \tilde{\boldsymbol{w}}_{1}^{H} \boldsymbol{h}_{2}}{\boldsymbol{h}_{1}^{H} \tilde{\boldsymbol{w}}_{1} \tilde{\boldsymbol{w}}_{1}^{H} \boldsymbol{h}_{1}}-\frac{\left(A_{1}^{\min }+1\right) \boldsymbol{h}_{2}^{H} \tilde{\boldsymbol{w}}_{2} \tilde{\boldsymbol{w}}_{2}^{H} \boldsymbol{h}_{2}}{\boldsymbol{h}_{1}^{H} \tilde{\boldsymbol{w}}_{2} \tilde{\boldsymbol{w}}_{2}^{H} \boldsymbol{h}_{1}}+1 \\
= & \frac{A_{1}^{\min } \boldsymbol{h}_{2}^{H} \boldsymbol{\Lambda} \boldsymbol{h}_{1} \boldsymbol{h}_{1}^{H} \boldsymbol{\Lambda}^{H} \boldsymbol{h}_{2}}{\boldsymbol{h}_{1}^{H} \boldsymbol{\Lambda} \boldsymbol{h}_{1} \boldsymbol{h}_{1}^{H} \boldsymbol{\Lambda}^{H} \boldsymbol{h}_{1}}-\frac{\left(A_{1}^{\min }+1\right) \boldsymbol{h}_{2}^{H} \boldsymbol{h}_{1} \boldsymbol{h}_{1}^{H} \boldsymbol{h}_{2}}{\boldsymbol{h}_{1}^{H} \boldsymbol{h}_{1} \boldsymbol{h}_{1}^{H} \boldsymbol{h}_{1}}+1 \\
= & \frac{A_{1}^{\min } \boldsymbol{h}_{2}^{H} \boldsymbol{h}_{1} \boldsymbol{h}_{1}^{H} \boldsymbol{h}_{2}}{\boldsymbol{h}_{1}^{H} \boldsymbol{h}_{1} \boldsymbol{h}_{1}^{H} \boldsymbol{h}_{1}}-\frac{\left(A_{1}^{\min }+1\right) \boldsymbol{h}_{2}^{H} \boldsymbol{h}_{1} \boldsymbol{h}_{1}^{H} \boldsymbol{h}_{2}}{\boldsymbol{h}_{1}^{H} \boldsymbol{h}_{1} \boldsymbol{h}_{1}^{H} \boldsymbol{h}_{1}}+1 \\
= & 1-\frac{\boldsymbol{h}_{2}^{H} \boldsymbol{h}_{1} \boldsymbol{h}_{1}^{H} \boldsymbol{h}_{2}}{\boldsymbol{h}_{1}^{H} \boldsymbol{h}_{1} \boldsymbol{h}_{1}^{H} \boldsymbol{h}_{1}}>0,
\end{aligned}
$$


Therefore, the constraint in (36) is not held. The case $\lambda_{1}>$ $0, \lambda_{2}>0, \lambda_{3}=0$ is also impossible. Therefore, the only possible case is $\lambda_{1}>0, \lambda_{2}=0, \lambda_{3}>0$.

3) The case of $\lambda_{1}>0, \lambda_{2}=0, \lambda_{3}>0$ : According to (83) and (84), we have

$$
\begin{gathered}
\boldsymbol{w}_{1}-\lambda_{1} \boldsymbol{h}_{1} \boldsymbol{h}_{1}^{H} \boldsymbol{w}_{1}+\frac{\lambda_{3}}{\sigma^{2}} \boldsymbol{h}_{2} \boldsymbol{h}_{2}^{H} \boldsymbol{w}_{1}=0 \\
\boldsymbol{w}_{2}-\frac{\lambda_{3}}{\sigma^{2} A_{2}^{\min }} \boldsymbol{h}_{2} \boldsymbol{h}_{2}^{H} \boldsymbol{w}_{2}=0
\end{gathered}
$$

therefore,

$$
\begin{aligned}
& \boldsymbol{w}_{1}=\left(\boldsymbol{I}_{T}+\frac{\lambda_{3}}{\sigma^{2}} \boldsymbol{h}_{2} \boldsymbol{h}_{2}^{H}\right)^{-1} \boldsymbol{h}_{1} \lambda_{1} \boldsymbol{h}_{1}^{H} \boldsymbol{w}_{1}, \\
& \boldsymbol{w}_{2}=\boldsymbol{h}_{2} \frac{\lambda_{3}}{\sigma^{2} A_{2}^{\min }} \boldsymbol{h}_{2}^{H} \boldsymbol{w}_{2} .
\end{aligned}
$$

According to Lemma $2, \lambda_{1} \boldsymbol{h}_{1}^{H} \boldsymbol{w}_{1}$ and $\frac{\lambda_{3}}{\sigma^{2} A_{2}^{\min }} \boldsymbol{h}_{2}^{H} \boldsymbol{w}_{2}$ can be both scalars and the optimal $\boldsymbol{w}_{1}$ and $\boldsymbol{w}_{2}$ must be respectively parallel to $\left(\boldsymbol{I}_{T}+\frac{\lambda_{3}}{\sigma^{2}} \boldsymbol{h}_{2} \boldsymbol{h}_{2}^{H}\right)^{-1} \boldsymbol{h}_{1}$ and $\boldsymbol{h}_{2}$. In the other words, the optimal beamforming vectors are $\boldsymbol{w}_{1}=\sqrt{p_{1}} \tilde{\boldsymbol{w}}_{1}, \boldsymbol{w}_{2}=$ $\sqrt{p_{2}} \tilde{\boldsymbol{w}}_{2}$, where

$$
\begin{aligned}
& \tilde{\boldsymbol{w}}_{1}=\frac{\left(\boldsymbol{I}_{T}+\frac{\lambda_{3}}{\sigma^{2}} \boldsymbol{h}_{2} \boldsymbol{h}_{2}^{H}\right)^{-1} \boldsymbol{h}_{1}}{\left|\left(\boldsymbol{I}_{T}+\frac{\lambda_{3}}{\sigma^{2}} \boldsymbol{h}_{2} \boldsymbol{h}_{2}^{H}\right)^{-1} \boldsymbol{h}_{1}\right|}, \\
& \tilde{\boldsymbol{w}}_{2}=\frac{\boldsymbol{h}_{2}}{\left|\boldsymbol{h}_{2}\right|},
\end{aligned}
$$

are the normalized beamforming directions for users with Lagrangian multiplier $\lambda_{3}=\sigma^{2} A_{2}^{\min } / \boldsymbol{h}_{2}^{H} \boldsymbol{h}_{2}$, which is obtained from (102). In addition, using (80) and (81) with $\lambda_{1}>0, \lambda_{3}>$ 0 , i.e.,

$$
\begin{gathered}
A_{1}^{\min } \sigma^{2}-p_{1} \boldsymbol{h}_{1}^{H} \tilde{\boldsymbol{w}}_{1} \tilde{\boldsymbol{w}}_{1}^{H} \boldsymbol{h}_{1}=0 \\
1 / \sigma^{2} \boldsymbol{h}_{2}^{H} \boldsymbol{w}_{1} \boldsymbol{w}_{1}^{H} \boldsymbol{h}_{2}+1-\frac{1}{\sigma^{2} A_{2}^{\min }} \boldsymbol{h}_{2}^{H} \boldsymbol{w}_{2} \boldsymbol{w}_{2}^{H} \boldsymbol{h}_{2}=0
\end{gathered}
$$

the optimal transmit powers are given as

$$
\begin{gathered}
p_{1}=\frac{A_{1}^{\min } \sigma^{2}}{\mathbf{h}_{1}^{H} \tilde{\boldsymbol{w}}_{1} \tilde{\boldsymbol{w}}_{1}{ }^{H} \mathbf{h}_{1}}, \\
p_{2}=\frac{\left(A_{1}^{\min } \mathbf{h}_{2}^{H} \tilde{\boldsymbol{w}}_{1} \tilde{\boldsymbol{w}}_{1}{ }^{H} \mathbf{h}_{2}+\mathbf{h}_{1}^{H} \tilde{\boldsymbol{w}}_{1} \tilde{\boldsymbol{w}}_{1}{ }^{H} \mathbf{h}_{1}\right) A_{2}^{\min } \sigma^{2}}{\mathbf{h}_{1}^{H} \tilde{\boldsymbol{w}}_{1} \tilde{\boldsymbol{w}}_{1}{ }^{H} \mathbf{h}_{1} \mathbf{h}_{2}^{H} \mathbf{h}_{2}} .
\end{gathered}
$$

Therefore, the optimal beamforming vectors are as in (42), which completes the proof.

\section{J. Proof of Corollary 3}

It was shown in the proof of Proposition 5 that the optimal beamforming is achieved for $\lambda_{1}>0, \lambda_{2}=0, \lambda_{3}>0$. Then, according to the DPC scheme formulated in (14), it is easy to find that the optimal beamforming vectors proposed in (5) achieves the same performance as DPC.

\section{K. Proof of Proposition 6}

First, we prove sufficiency of the condition. If condition C3 is satisfied, according to the proof of Proposition 5, we find that the relaxed constraint, i.e., (36), can be also satisfied, implying the optimal solution can satisfy all the proposed constraints in problem (27). Therefore, with condition C3, the optimal beamforming proposed in Proposition 5 can be achieved.

To prove the necessity of the condition, we note that in the proof of Proposition 5, only in the case of $\lambda_{1}>0, \lambda_{2}=$ $0, \lambda_{3}>0$, we can achieve the optimal solution to (27). In this case, the constraint (36) is relaxed, hence the optimal solution should satisfy this constraint, i.e.,

$$
1 / \sigma^{2} \mathbf{h}_{1}^{H} \boldsymbol{w}_{1}^{*} \boldsymbol{w}_{1}^{* H} \mathbf{h}_{1}+1-\frac{1}{\sigma^{2} A_{2}^{\min }} \mathbf{h}_{1}^{H} \boldsymbol{w}_{2}^{*} \boldsymbol{w}_{2}^{* H} \mathbf{h}_{1} \leq 0
$$

which is in fact condition $\mathrm{C} 3$ if the optimal beamforming vectors proposed in Proposition 5 are substituted.

\section{REFERENCES}

[1] J. G. Andrews, S. Buzzi, W. Choi, S. V. Hanly, A. Lozano, A. C. K. Soong, and J. C. Zhang, "What will 5G be?", IEEE J. Sel. Areas Commun., vol. 32, pp. 1065-1082, Jun. 2014.

[2] S. He, Y. Huang, J. Wang, L. Yang, and W. Hong, "Joint antenna selection and energy-efficient beamforming design," IEEE Signal Process. Lett., vol. 23, pp. 1165-1169, Sep. 2016.

[3] J. Wang, W. Guan, Y. Huang, R. Schober, and X. You, "Distributed optimization of hierarchical small cell networks: A GNEP framework," IEEE J. Sel. Areas Commun., vol. 35, pp. 249-264, Feb. 2017.

[4] X. Wang and X. Zhang, "Linear transmission for rate optimization in MIMO broadcast channels," IEEE Trans. Wireless Commun., vol. 9, pp. 3247-3257, Oct. 2010.

[5] H. Dai, Y. Huang, Y. Xu, C. Li, B. Wang, and L. Yang, "Energyefficient resource allocation for energy harvesting-based device-to-device communication," IEEE Trans. Veh. Technol., pp. 1-1, Nov. 2018.

[6] D. W. K. Ng, E. S. Lo, and R. Schober, "Dynamic resource allocation in MIMO-OFDMA systems with full-duplex and hybrid relaying," IEEE Trans. on Commun., vol. 60, pp. 1291-1304, May 2012.

[7] X. Chen, L. Wu, Z. Zhang, J. Dang, and J. Wang, "Adaptive modulation and filter configuration in universal filtered multi-carrier systems," IEEE Trans. on Wireless Commun., vol. 17, pp. 1869-1881, Mar. 2018.

[8] A. Wiesel, Y. C. Eldar, and S. Shamai, "Linear precoding via conic optimization for fixed MIMO receivers," IEEE Trans. on Signal Process., vol. 54, pp. 161-176, Jan. 2006.

[9] Q. H. Spencer, A. L. Swindlehurst, and M. Haardt, "Zero-forcing methods for downlink spatial multiplexing in multiuser MIMO channels," IEEE Trans. on Signal Process., vol. 52, pp. 461-471, Feb. 2004.

[10] H. Weingarten, Y. Steinberg, and S. S. Shamai, "The capacity region of the gaussian multiple-input multiple-output broadcast channel," IEEE Trans. Inform. Theory, vol. 52, pp. 3936-3964, Sep. 2006.

[11] Z. Chen, Z. Ding, P. Xu, and X. Dai, "Optimal precoding for a QoS optimization problem in two-user MISO-NOMA downlink," IEEE Commun. Lett., vol. 20, pp. 1263-1266, Jun. 2016.

[12] L. Dai, B. Wang, Y. Yuan, S. Han, C. I, and Z. Wang, "Non-orthogonal multiple access for 5G: solutions, challenges, opportunities, and future research trends," IEEE Commun. Mag., vol. 53, pp. 74-81, Sep. 2015.

[13] Z. Ding, X. Lei, G. K. Karagiannidis, R. Schober, J. Yuan, and V. K. Bhargava, "A survey on non-orthogonal multiple access for 5G networks: Research challenges and future trends," IEEE J. Sel. Areas Commun., vol. 35, pp. 2181-2195, Oct. 2017.

[14] X. Sun, S. Yan, N. Yang, Z. Ding, C. Shen, and Z. Zhong, "Shortpacket downlink transmission with non-orthogonal multiple access," IEEE Trans. on Wireless Commun., vol. 17, pp. 4550-4564, Jul. 2018.

[15] Y. Feng, S. Yan, and Z. Yang, "Secure transmission to the strong user in non-orthogonal multiple access," IEEE Commun. Lett., vol. 22, pp. 2623-2626, Dec. 2018.

[16] Y. Huang, C. Zhang, J. Wang, Y. Jing, L. Yang, and X. You, "Signal processing for MIMO-NOMA: Present and future challenges," IEEE Wireless Commun., vol. 25, pp. 32-38, Apr. 2018. 
[17] Z. Ding, F. Adachi, and H. V. Poor, "The application of MIMO to nonorthogonal multiple access," IEEE Trans. on Wireless Commun., vol. 15, pp. 537-552, Jan. 2016.

[18] X. Sun, N. Yang, S. Yan, Z. Ding, D. W. K. Ng, C. Shen, and Z. Zhong, "Joint beamforming and power allocation in downlink NOMA multiuser MIMO networks," IEEE Trans. on Wireless Commun., vol. 17, pp. 53675381, Aug. 2018.

[19] Y. Sun, D. W. K. Ng, J. Zhu, and R. Schober, "Robust and secure resource allocation for full-duplex MISO multicarrier NOMA systems," IEEE Trans. on Commun., vol. 66, pp. 4119-4137, Sep. 2018.

[20] Y. Feng, S. Yan, Z. Yang, N. Yang, and J. Yuan, "Beamforming design and power allocation for secure transmission with NOMA," IEEE Trans. on Wireless Commun., vol. 18, pp. 2639-2651, May 2019.

[21] F. Fang, H. Zhang, J. Cheng, and V. C. M. Leung, "Energy-efficient resource allocation for downlink non-orthogonal multiple access network," IEEE Trans. on Commun., vol. 64, pp. 3722-3732, Sep. 2016.

[22] K. Wang, J. Cui, Z. Ding, and P. Fan, "Stackelberg game for user clustering and power allocation in millimeter wave-NOMA systems," IEEE Trans. on Wireless Commun., vol. 18, pp. 2842-2857, May 2019.

[23] J. Zhu, J. Wang, Y. Huang, S. He, X. You, and L. Yang, "On optimal power allocation for downlink non-orthogonal multiple access systems," IEEE J. Sel. Areas Commun., vol. 35, pp. 2744-2757, Dec. 2017.

[24] J. Cui, Z. Ding, and P. Fan, "Beamforming design for MISO nonorthogonal multiple access systems," IET Commun., vol. 11, pp. 720725, Apr. 2017.

[25] F. Zhu, Z. Lu, J. Zhu, J. Wang, and Y. Huang, "Beamforming design for downlink non-orthogonal multiple access systems," IEEE Access, vol. 6, pp. 10956-10965, Jan. 2018

[26] M. F. Hanif, Z. Ding, T. Ratnarajah, and G. K. Karagiannidis, "A minorization-maximization method for optimizing sum rate in the downlink of non-orthogonal multiple access systems," IEEE Trans. on Signal Process., vol. 64, pp. 76-88, Jan. 2016.

[27] Y. Sun, D. W. K. Ng, J. Zhu, and R. Schober, "Robust and secure resource allocation for full-duplex MISO multicarrier NOMA systems," IEEE Trans. on Commun., vol. 66, pp. 4119-4137, Sep. 2018.

[28] F. Alavi, K. Cumanan, Z. Ding, and A. G. Burr, "Beamforming techniques for nonorthogonal multiple access in $5 \mathrm{G}$ cellular networks," IEEE Trans. on Veh. Technol., vol. 67, pp. 9474-9487, Oct. 2018.

[29] J. Choi, "Minimum power multicast beamforming with superposition coding for multiresolution broadcast and application to NOMA systems," IEEE Trans. on Commun., vol. 63, pp. 791-800, Mar. 2015.

[30] J. Choi, "A suboptimal approach for minimum transmit power NOMA beamforming," in Proc. IEEE Veh. Technol. Conf., pp. 1-5, Sep. 2017.

[31] Z. Chen, Z. Ding, X. Dai, and G. K. Karagiannidis, "On the application of quasi-degradation to MISO-NOMA downlink," IEEE Trans. on Signal Process., vol. 64, pp. 6174-6189, Dec. 2016.

[32] Y. Liu, H. Xing, C. Pan, A. Nallanathan, M. Elkashlan, and L. Hanzo, "Multiple-antenna-assisted non-orthogonal multiple access," IEEE Wireless Commun., vol. 25, pp. 17-23, Apr. 2018.

[33] Y. Huang and D. P. Palomar, "Rank-constrained separable semidefinite programming with applications to optimal beamforming," IEEE Trans. on Signal Process., vol. 58, pp. 664-678, Feb. 2010.

[34] C. B. Peel, B. M. Hochwald, and A. L. Swindlehurst, "A vector-perturbation technique for near-capacity multiantenna multiuser communication-part i: channel inversion and regularization," IEEE Trans. on Commun., vol. 53, pp. 195-202, Jan. 2005.

[35] E. Bjornson, M. Bengtsson, and B. Ottersten, "Optimal multiuser transmit beamforming: A difficult problem with a simple solution structure [lecture notes]," IEEE Signal Process. Mag., vol. 31, pp. 142-148, Jul. 2014.

[36] W. Yu and T. Lan, "Transmitter optimization for the multi-antenna downlink with per-antenna power constraints," IEEE Trans. Signal Process., vol. 55, pp. 2646-2660, Jun. 2007.

[37] Z. Luo, W. Ma, A. M. So, Y. Ye, and S. Zhang, "Semidefinite relaxation of quadratic optimization problems," IEEE Signal Process. Mag., vol. 27, pp. 20-34, May 2010.

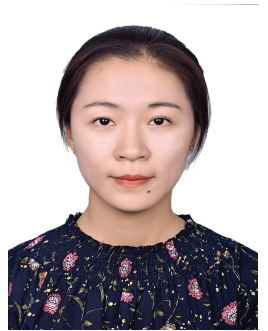

Jianyue Zhu (S'17) received the B.S. degree from Nanjing Agricultural University in 2015. She is currently working towards the Ph.D. degree in information and communication engineering at the School of Information Science and Engineering, Southeast University, Nanjing, China. During 20182019, Dr. Zhu was visiting the School of Computing and Communications, Lancaster University, UK. Her current research interests include non-orthogonal multiple access, intelligient reflecting surface, and optimization theory.

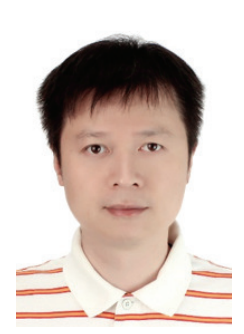

Jiaheng Wang (M'10-SM'14) received the Ph.D. degree in electronic and computer engineering from the Hong Kong University of Science and Technology, Kowloon, Hong Kong, in 2010, and the B.E. and M.S. degrees from the Southeast University, Nanjing, China, in 2001 and 2006, respectively.

$\mathrm{He}$ is currently a Full Professor at the National Mobile Communications Research Laboratory (NCRL), Southeast University, Nanjing, China. From 2010 to 2011, he was with the Signal Processing Laboratory, KTH Royal Institute of Technology, Stockholm, Sweden. He also held visiting positions at the Friedrich Alexander University Erlangen-NÃđErnberg, NÃđErnberg, Germany, and the University of Macau, Macau. His research interests include optimization in signal processing and wireless communications.

Dr. Wang has published more than 100 articles on international journals and conferences. From 2014 to 2018, he served as an Associate Editor for the IEEE Signal Processing Letters. From 2018, he serves as a Senior Area Editor for the IEEE Signal Processing Letters. He was a recipient of the Humboldt Fellowship for Experienced Researchers and the best paper awards of IEEE GLOBECOM 2019, ADHOCNETS 2019, and WCSP 2014.

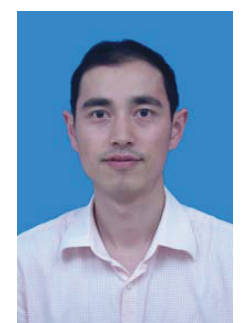

Yongming Huang (M'10-SM'17) received the B.S. and M.S. degrees from Nanjing University, China, in 2000 and 2003, respectively. In 2007 he received the $\mathrm{Ph} . \mathrm{D}$. degree in electrical engineering from Southeast University, China. Since March 2007 he has been a faculty in the School of Information Science and Engineering, Southeast University, China, where he is currently a full professor. During 2008-2009, Dr. Huang was visiting the Signal Processing Lab, Electrical Engineering, Royal Institute of Technology $(\mathrm{KTH})$, Stockholm, Sweden.

His current research interests include MIMO wireless communications, millimeter wave wireless communications and Intelligent mobile communications. He has published over 200 peer-reviewed papers, hold over 80 invention patents. He submitted around 20 technical contributions to IEEE standards, and was awarded a certificate of appreciation for outstanding contribution to the development of IEEE standard 802.11aj. He has served as an Associate Editor for the IEEE Transactions on Signal Processing, IEEE Wireless Communications Letters, EURASIP Journal on Advances in Signal Processing, and EURASIP Journal on Wireless Communications and Networking, and a guest editor for the IEEE Journal Selected Areas in Communications. 


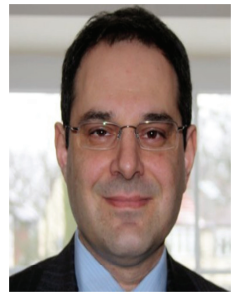

Keivan Navaie (S'00-M'04-SM'10) is with the School of Computing and Communications, Lancaster University, UK. His research seeks innovative inter-disciplinary solutions to address fundamental technical and design challenges in Intelligent Cyber Physical Systems and their integrations into dayto-day life to improve its sustainability and antifragility. Keivan's research covers topics including Distributed Cloud and Edge Computing, Connectivity Resilience in Cyber-Physical Systems and IoT, Applications of Machine Learning and Artificial Intelligence, and Cognitive Communications Networks. He is on the editorial board of the IEEE Comm. Letters, IEEE Comm. Surveys and Tutorials, Trans. on Wireless Communications, and IEEE Access. Keivan is also advocate of evidence-based policy making through his involvement with the EU Environment Agency and EU Parliament as an Independent Scientific Expert. $\mathrm{He}$ is a Senior member of IEEE, a Fellow of IET and a Chartered Engineer in the UK.

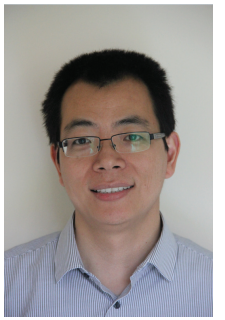

Zhiguo Ding (S'03-M'05-F'20) received his B.Eng in Electrical Engineering from the Beijing University of Posts and Telecommunications in 2000, and the $\mathrm{Ph} . \mathrm{D}$ degree in Electrical Engineering from Imperial College London in 2005. From Jul. 2005 to Apr. 2018, he was working in Queen's University Belfast, Imperial College, Newcastle University and Lancaster University. Since Apr. 2018, he has been with the University of Manchester as a Professor in Communications. From Oct. 2012 to Sept. 2020, he has also been an academic visitor in Princeton

University.

Dr Ding' research interests are 5G networks, game theory, cooperative and energy harvesting networks and statistical signal processing. He is serving as an Area Editor for the IEEE Open Journal of the Communications Society, an Editor for IEEE Transactions on Communications, IEEE Transactions on Vehicular Technology, and Journal of Wireless Communications and Mobile Computing, and was an Editor for IEEE Wireless Communication Letters, IEEE Communication Letters from 2013 to 2016. He received the best paper award in IET ICWMC-2009 and IEEE WCSP-2014, the EU Marie Curie Fellowship 2012-2014, the Top IEEE TVT Editor 2017, IEEE Heinrich Hertz Award 2018, IEEE Jack Neubauer Memorial Award 2018 and IEEE Best Signal Processing Letter Award 2018.

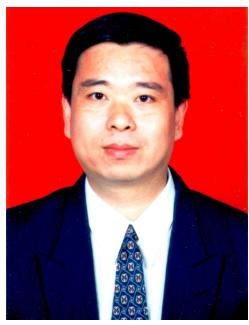

Luxi Yang (M'96) received the M.S. and Ph.D. degree in electrical engineering from the Southeast University, Nanjing, China, in 1990 and 1993, respectively. Since 1993, he has been with the Department of Radio Engineering, Southeast University, where he is currently a full professor of information systems and communications, and the Director of Digital Signal Processing Division. His current research interests include signal processing for wireless communications, MIMO communications, cooperative relaying systems, and statistical signal processing. He has authored or co-authored of two published books and more than 100 journal papers, and holds 30 patents. Prof. Yang received the first and second class prizes of science and technology progress awards of the state education ministry of China in 1998, 2002 and 2014. He is currently a member of Signal Processing Committee of Chinese Institute of Electronics. 